\title{
Using a Geographic Information System and Scanning Technology to Create High-resolution Land-use Data Sets
}

\section{U.S. GEOLOGICAL SURVEY}

Water-Resources Investigations Report 96-4100
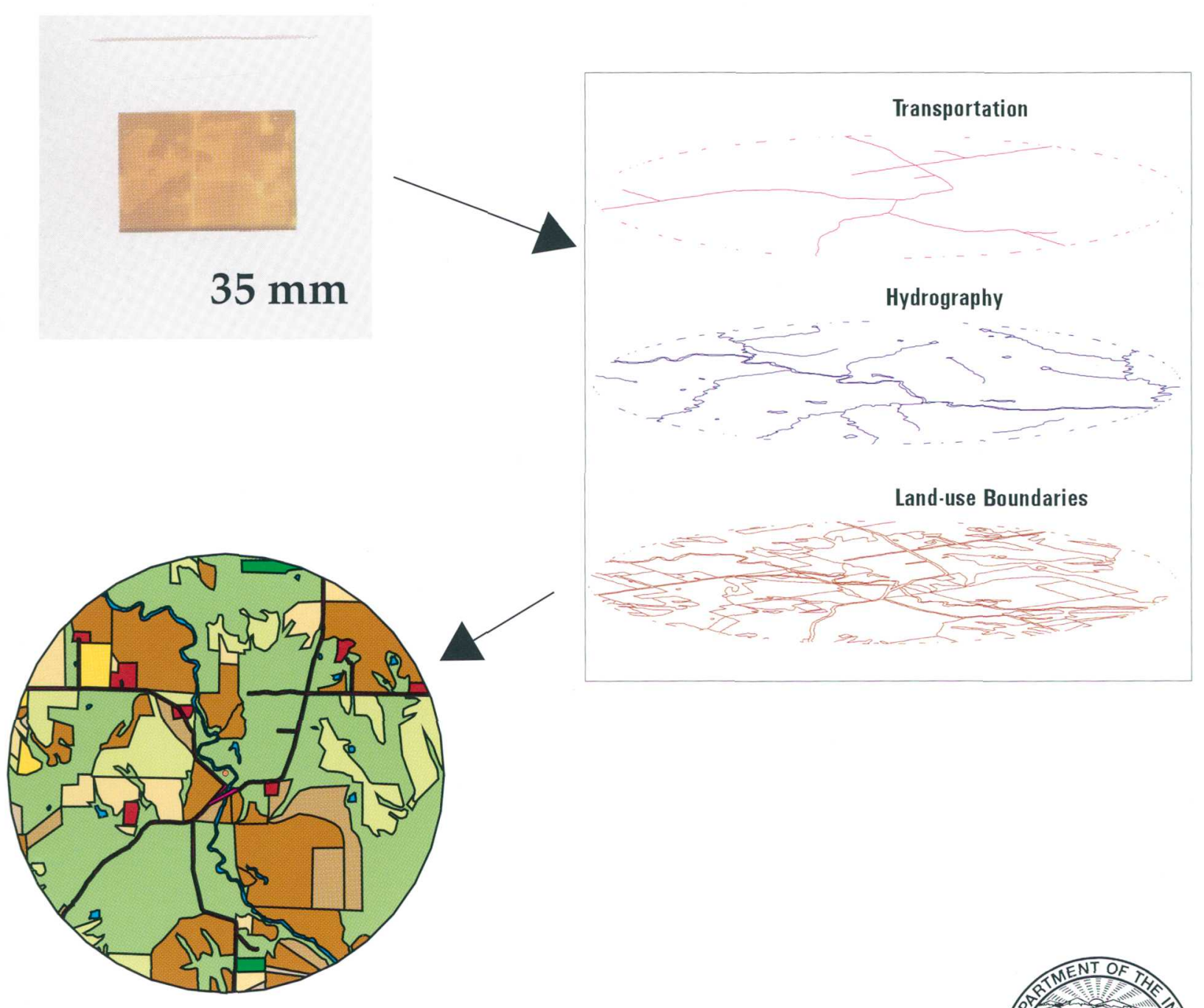

Prepared in cooperation with the U.S. Environmental Protection Agency

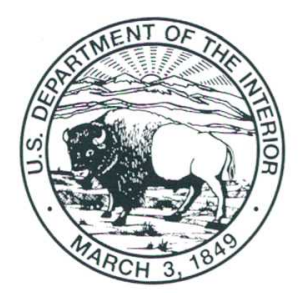




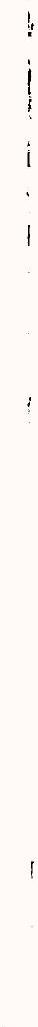




\section{Using a Geographic Information System and Scanning Technology to Create High-resolution Land-use Data Sets}

By CRAIG A. HARVEY, DANA W. KOLPIN, and WILLIAM A. BATTAGLIN

\section{U.S. GEOLOGICAL SURVEY}

Water-Resources Investigations Report 96-4100

Prepared in cooperation with the

U.S. Environmental Protection Agency

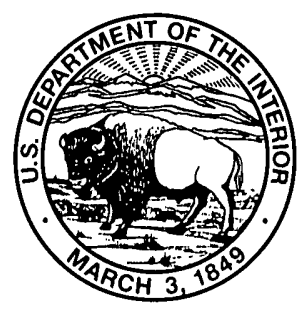




\title{
U.S. DEPARTMENT OF THE INTERIOR \\ BRUCE BABBITT, Secretary
}

\author{
U.S. GEOLOGICAL SURVEY
}

Gordon P. Eaton, Director

The use of firm, trade, and brand names in this report is for identification purposes only and does not constitute endorsement by the U.S. Geological Survey.

For additional information write to:

District Chief

U.S. Geological Survey

Rm. 269, Federal Building

400 South Clinton Street

lowa City, IA 52244
Copies of this report can be purchased from:

U.S. Geological Survey Branch of Information Services

Box 25286

Federal Center

Denver, CO 80225 


\section{CONTENTS}

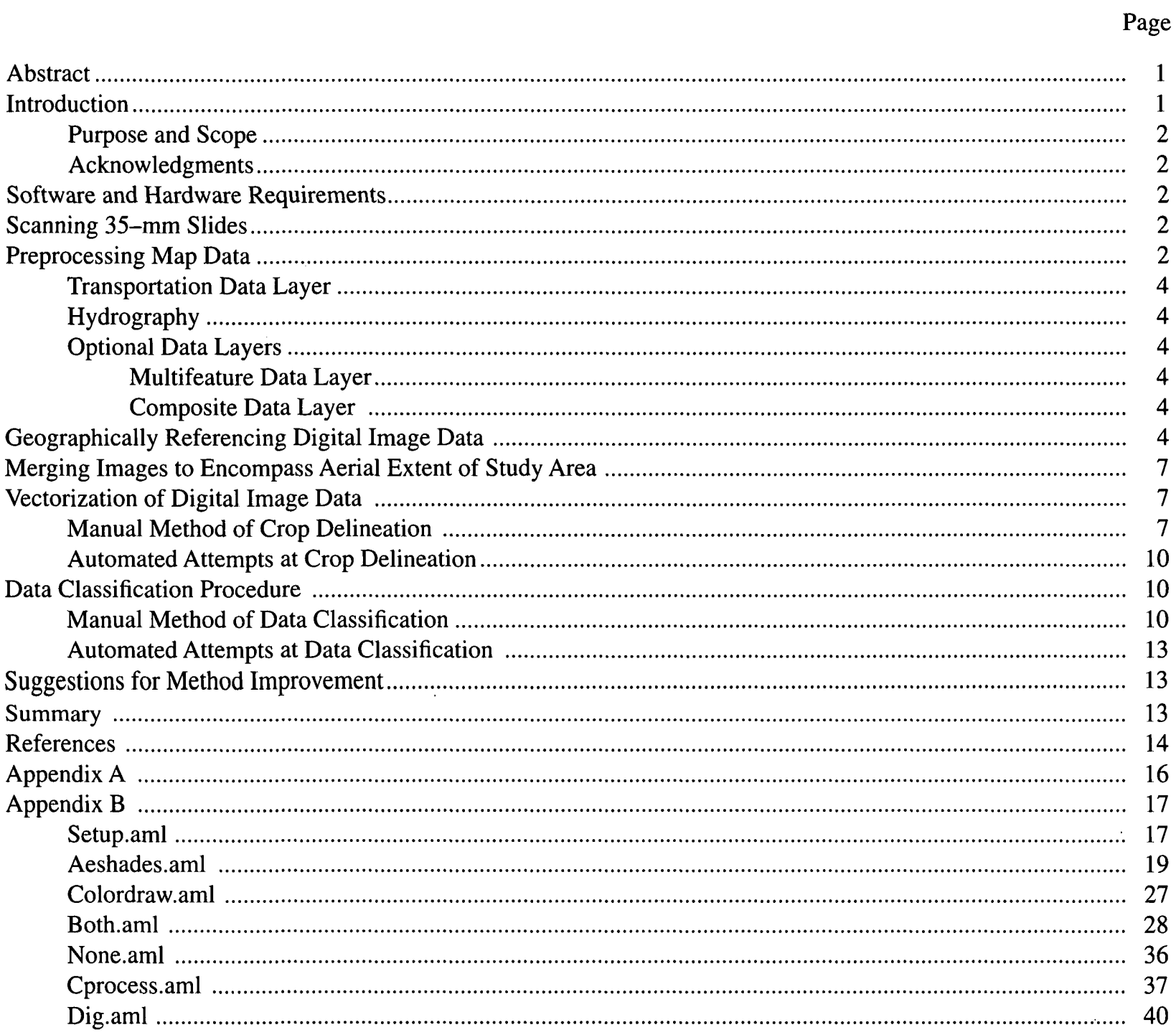

\section{ILLUSTRATIONS}

Figure 1. Source-data layers used for land-use classification, shown overlaid on the 2-kilometer buffer area-

(A) Transportation data layer, (B) Hydrography data layer, (C) Well location

2. $\boldsymbol{A}$ Diagrams showing an example of using a GIS data layer to graphically register a raster image

$\boldsymbol{B}$ Diagram showing an example of registration interface

3. Example of transportation and hydrography data layers overlaid on a registered and rectified composite color image

4. Example of field delineations overlaid on a registered and rectified composite color image.

5. Examples of data classified using Consolidated Farm Services Agency (CFSA) crop maps and unclassified areas representing undocumented classifications in the CFSA crop data

6. Example of data classification completed using Consolidated Farm Services Agency (CFSA) crop maps and classification using areas with documented classification as visual training areas 


\begin{tabular}{rll}
\hline Multiply & By & To obtain \\
\hline foot $(\mathrm{ft})$ & 0.3048, & meter \\
mile $(\mathrm{mi})$ & 1.609 & kilometer \\
square mile $\left(\mathrm{mi}^{2}\right)$ & 2.590 & square kilometer \\
foot per mile $\left(\mathrm{ft} / \mathrm{mi}^{2}\right)$ & 0.1894 & meter per kilometer \\
mile per square $\mathrm{mile}\left(\mathrm{mi} / \mathrm{mi}^{2}\right)$ & 0.621 & kilometer per square kilometer \\
\hline
\end{tabular}

Sea level: In this report, "sea level" refers to the National Geodetic Vertical Datum of 1929 (NGVD of 1929) - a geodetic datum derived from a general adjustment of the first-order level nets of both the United States and Canada, formerly called Sea Level Datum of 1929.

\section{DEFINITION OF TERMS}

AML_ARC Macro Language, a post-processing language written for use with ARC/INFO.

Append (appending) — the act of merging adjacent or overlapping digital data layers.

ARC - geographic information system (GIS) licensed through Environmental Systems Research Institute, Inc.

ARC/EDIT - geographic information system (GIS) edit module licensed through Environmental Systems Research Institute, Inc.

ARC/GRID—geographic information system (GIS) raster modeling module licensed through Environmental Systems Research Institute, Inc.

ARC/INFO—geographic information system (GIS) relational database software licensed through Environmental Systems Research Institute, Inc.

ARC/PLOT-geographic information system (GIS) plotting module licensed through Environmental Systems Research Institute, Inc.

Attributes-numeric or text characteristics that describe a specific spatial feature; attributes are commonly stored in a relational database structure (Nebert, 1989).

Clip-to reduce the areal extent of a coverage to match the areal extent of a different coverage.

Coverage (cover) - a digital data set depicting a defined spatial extent composed of one or more data layers.

DLG-digital line graph data, a file of digital point coordinates representing linear features of data layers such as hydrography, hypsography, transportation, stored in vector format and organized by quadrangles.

Hydrography—surface-water drainage network.

Hypsography—elevation contours.

Image registration-image data, in page units, transformed into a geographically referenced coordinate system using a series of at least 4 identifiable features in the image and an existing vector data set.

INFO-item-the name of an INFO-data-file item. Info items can have values attributed to them.

Lattice—raster data stored in an ARC/INFO grid format.

Raster data-data that are maintained in the form of an array. The data are cell-based.

Register-data transformed from a simple coordinate system into a geographically referenced coordinate system.

Source-data layer-digital data layers required to complete a particular process.

Topology - the explicit connectivity of spatial features. Topology is required in an arc-node data model (such as digital line graphs) for proper associations to be made among features (such as arcs, nodes, points, and polygons) and their attributes (Nebert, 1989).

Vector data-data that are maintained in the form of points, lines, or polygons that are defined by a set of cartesian coordinates (Cartier and others, 1993, p. 4). 


\title{
Using a Geographic Information System and Scanning Technology to Create High-Resolution Land-Use Data Sets
}

\author{
By Craig A. Harvey, Dana W. Kolpin, and William A. Battaglin
}

\section{ABSTRACT}

A geographic information system (GIS) procedure was developed to compile low-altitude aerial photography, digitized data, and land-use data from U.S. Department of Agriculture Consolidated Farm Service Agency (CFSA) offices into a high-resolution (approximately 5 meters) land-use GIS data set. The aerial photography consisted of 35-mm slides which were scanned into tagged information file format (TIFF) images. These TIFF images were then imported into the GIS where they were registered into a geographically referenced coordinate system. Boundaries between land use were delineated from these GIS data sets using on-screen digitizing techniques. Crop types were determined using information obtained from the U.S. Department of Agriculture CFSA offices. Crop information not supplied by the CFSA was attributed by manual classification procedures. Automated methods to provide delineation of the field boundaries and land-use classification were investigated. It was determined that using these data sources, automated methods were less efficient and accurate than manual methods of delineating field boundaries and classifying land use.

\section{INTRODUCTION}

Since 1991, the U.S. Geological Survey(USGS) has been investigating the regional occurrence of agricultural chemicals in near-surface aquifers of the midwestern United States (Kolpin and Burkart, 1991; Kolpin and others, 1993; Kolpin and others, 1994;
Kolpin and Thurman, 1995; Kolpin and others, 1995). One objective of this effort was to determine possible effects land use may have on the occurrence of agricultural chemicals in ground water. Although several relations to land use were identified (Kolpin and others, 1994), it was felt that the analysis was limited because the information was based on broad visual estimations and the unavoidable inconsistency inherent when multiple personnel are providing the descriptions of land use. To better understand the relation between land use and the occurrence of agricultural chemicals in ground water, more detailed land-use information surrounding the sampled wells was required.

A cooperative study was done with the U.S. Environmental Protection Agency from 1993 to 1994 to determine high-resolution land use within a 2-kilometer-radius spatial buffer surrounding selected wells. However, there are few data sources available from which to obtain historical land-use information over large areas. Although available satellite data has widespread coverage, it is expensive to obtain, requires land-use classification, and is of insufficient resolution to be used for this study. Another source of historical land-use information is from the U.S. Department of Agriculture (USDA), Consolidated Farm Service Agency (CFSA), county offices (formerly known as the Agriculture Stabilization and Conservation Service). The CFSA obtains low-altitude photography on an annual basis that can be obtained as $35-\mathrm{mm}$ color slides. Unlike satellite data, classification is not necessary when using CFSA photography because data on crops and management practices can also be obtained from the CFSA. The CFSA photography also is available at a lower cost and higher resolution than the available satellite data. Consequently, CFSA photography 
was utilized for this study to obtain detailed land-use data for areas surrounding selected wells.

\section{Purpose and Scope}

The purpose of this report is to describe the procedure developed to transfer the CFSA land-use data into a usable high-resolution GIS data base. This description will include hardware and software requirements, required data preprocessing, scanning and geographic information system (GIS) procedures implemented, and a description of the final land-use classification GIS data set produced using this method. The utility ARC/INFO Macro Language (AML) programs developed for use with this study are described in Appendix A and listed in Appendix B.

\section{Acknowledgments}

The authors thank the Consolidated Farm Services Agency offices contacted during this study for their cooperation in the timely delivery of both the 35 mm photography and the associated crop maps.

\section{SOFTWARE AND HARDWARE REQUIRE- MENTS}

Although many software and hardware combinations can be used to create GIS coverages of land use from 35-mm slide photography, the combination of software and hardware used for this study has demonstrated compatibility. The approach required the use of ARC/INFO version 6.0.1 or higher for Unix workstations, Adobe Photoshop for IBM compatible PC or Macintosh computers, and a communications package that provides for file transfer between systems.

The computer hardware used to complete this study included a Data General Avion 530 Unix workstation with 1.8 gigabytes of disk space and 128 megabytes of random access memory(RAM), a Power Macintosh 7100/66 AV with a 480 megabyte hard drive and 16 megabytes of RAM, and a Kodak Rapid Film Scanner 35 (RFS-35).

\section{SCANNING 35-MM SLIDES}

The scanning of 35-mm slides was tested using two methods. The first method attempted used Adobe
Photoshop on a $486 \mathrm{PC}$ to interface with the film scanner. However this method proved inefficient because of slow scanning times (about 10 minutes per slide) and limited capabilities of image manipulation. A subsequent, and more efficient method, used Adobe Photoshop on a Power Macintosh 7100/66 AV to interface with the film scanner. This method provided rapid slide scanning (about 2 minutes per slide) and versatile image manipulation.

The process for imaging slides begins with ensuring that power to the RFS-35 scanner is on before the computer is booted. This is necessary for the computer to recognize the scanner. The computer is then switched on and the system is booted, Adobe Photoshop is then initiated. The scanner is calibrated automatically each time it is powered up. Adobe Photoshop will post a window which prompts the user to empty the slide carriage and queries the user when that task is complete. Adobe Photoshop software will display the WAIT icon until the scanner calibration is complete.

Within the Photoshop window, starting at the menu bar (top), use the mouse to select the "File", the "Acquire", and the "Kodak 2035 RFS" options (Adobe Systems Incorporated, 1993). Next select the focus button followed by the prescan button. "Click the rectangular marque in the toolbox. Position the pointer at the upper left corner of the area of interest. A cross-hair pointer appears; while holding down the mouse button, drag the cursor diagonally to the lower right corner of the area of interest. Release the mouse button" (Adobe System Incorporated, 1993). A dashed white line indicates the areal extent of the image which will be scanned. Select the resolution at which the image is to be scanned; in this study 1,000 dots per inch (dpi) was used. Select the scan button; the scanned image will appear in a new window. The color saturation, as well as the contrast levels, can be manipulated by selecting "Image" (on the command bar, top) and making use of the listed functions. Save the image as a Macintosh tagged information format file (TIFF) with no compression.

\section{PREPROCESSING MAP DATA}

The method of land-use classification described herein requires several source data layers-a digital data layer of the transportation network (fig. 1A), a digital hydrography network (fig. 1B), and a well location centered within a user defined spatial-buffer area (fig. 1C). A 2-kilometer-radius spatial buffer, created using 
A

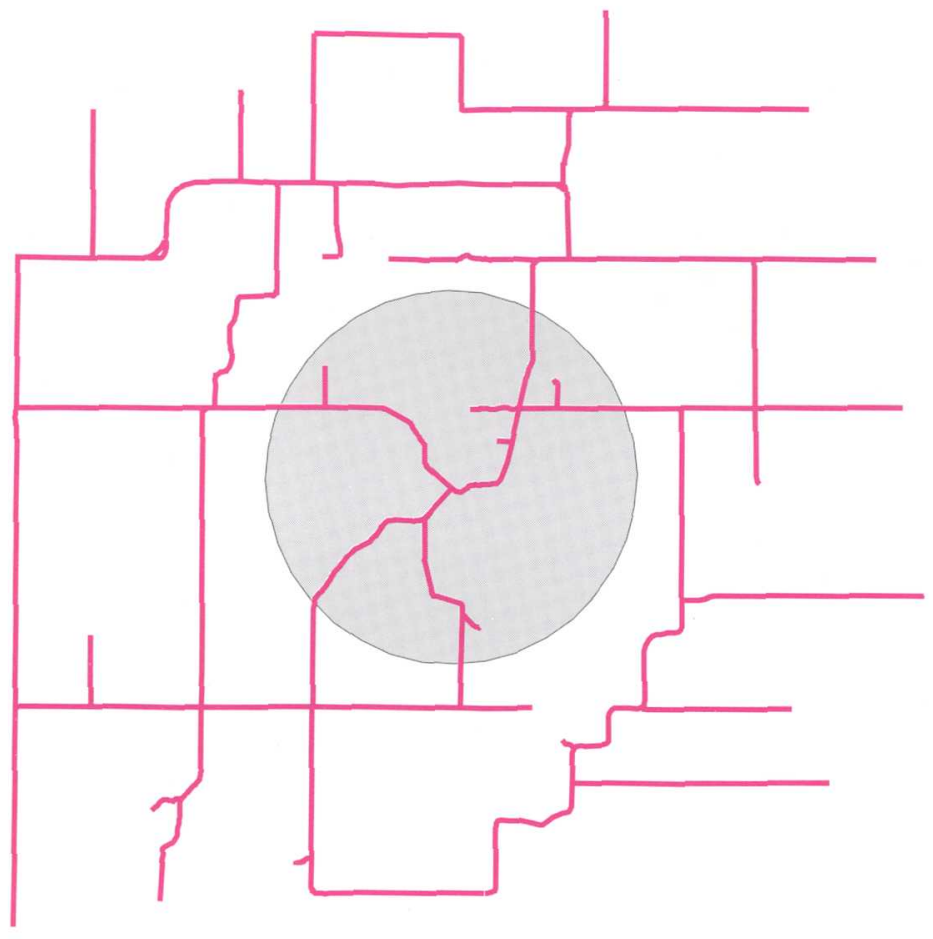

B

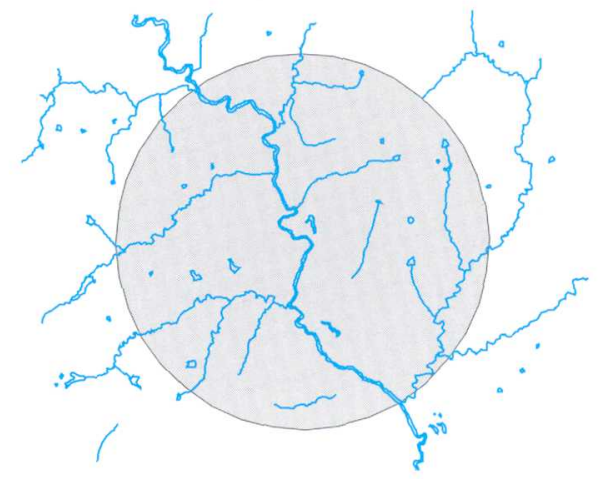

\section{EXPLANATION}

C

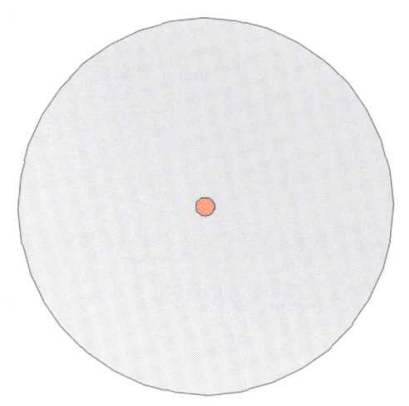

Spatial buffer (2-kilometer radius)

Roads

Surface Water

- Well

$\begin{array}{lllll}0 & 1 & 2 & 3 & \text { Kilometers }\end{array}$

Figure 1. Source-data layers used for land-use classification, shown overlaid on the 2-kilometer buffer area(A) Transportation data layer, (B) Hydrography data layer, (C) Well location. 
the ARC command BUFFER, was used for this study (fig. 1C). Optionally, combinations of the source data layers may prove useful in certain instances. These data layers are used to ensure proper registration of scanned images.

The data layers listed above can be clipped from a larger, available data set, scanned from mylar map separates, or digitized from hard-copy maps. For this study, source data were digitized from 1:24,000-scale USGS quadrangle maps. During manual digitization, maps were registered before digitizing their features. If necessary and when possible, the maps were reregistered until the root mean square (RMS) error of the transformation was equal to or less than 0.003 in digitizer inches (approximately 0.08 millimeters) (Cartier and others, 1993, p. 13). Source-data layers were created using standard digitizing commands within $\mathrm{ARC} /$ INFO. An area extending approximately 1.5 kilometers beyond the 2-kilometer-radius spatial buffer area was also digitized (fig. 1A.). This extended area was necessary to facilitate the location of image data with respect to the source data during the image registration phase of the study.

\section{Transportation Data Layer}

Roads and trails are digitized using standard digitizing techniques. Center lines are digitized for roads depicted on the map by two lines (fig. 1A). A typical paved width of a county road in this study was 22 to 24 feet plus as much as 10 feet of road shoulder. Therefore, the transportation data layer was buffered using a width of 33 feet representing 23 feet of road surface and 10 feet of road shoulder surface. This buffering is needed to develop a single polygon for roads that will be used as the template for the delineation of the field boundaries. The polygon is later identified as a road during the classification phase of this method.

\section{Hydrography}

Rivers, streams, and lakes are digitized using standard digitizing techniques. Rivers and streams which are depicted on the map as having two shores are digitized as such (fig. 1B). Lakes which have high and low levels depicted on the map need both shore lines digitized. Rivers and streams with two shores will be closed to form a single polygon, allowing for areal quantification in the final data layer.

\section{Optional Data Layers}

The optional data layers were produced only when needed to facilitate registration of an image. Typically the transportation data layer would suffice for registration, however there were areas which did not have roads or trails. In these instances, combinations of the transportation data layer and the hydrography data layer were used. In the most difficult cases, a multifeature data layer was generated and used in combination with all of the source-data layers.

\section{Multifeature Data Layer}

A multifeature data layer containing prominent features visible on the map, not included in the transportation or hydrography data layers, includes features that would be visible from an airplane and easily discernible on an aerial photograph (such as a high school track, an airstrip, or dams).

\section{Composite Data Layer}

This data layer is created by appending sourcedata layers together. The hydrography data layer may be composited with the transportation data layer and used to aid image registration. For images that are difficult to register, it may be advantageous to have prominent features delineated as a multifeatured data layer. The multifeature data layer may then be composited with the transportation data layer and/or the hydrography data layer to provide sufficient data for image registration. The data layer composed of several independent data layers is called the composite layer. Features which tend to have stable locations through time such as bridges, intersections of streams with roads, and dams, are particularly useful for image registration. However, when limited data are available, image registration by trial and error may be the only course available.

\section{GEOGRAPHICALLY REFERENCING DIGI- TAL IMAGE DATA}

The scanned 35-mm slides saved as TIFF images must be geographically referenced using the transportation, hydrography, and/or the optional data sets before conversion to ARC/INFO's grid format (fig. 2A). The ARC command REGISTER was used to provide image registration. Upon issuing the register command, four windows are created (fig. 2B). The window 
titled "OVERLAY" displays the registration coverage (transportation data layer) overlaid on the image (fig. 2B). The window titled "IMAGE" displays the image to be registered and the window titled "COVERAGE" displays the registration coverage (transportation data layer) (fig. 2B). A view box in both the COVERAGE and IMAGE windows depicts the areal extent to be overlaid in the OVERLAY window. Normally the areal extent of the coverage will be larger than that of the image. The view boxes can be moved and sized using various button combinations described in the ARC REFERENCE MANUAL (Environmental Systems Research Institute, 1994) or in the on-line help available with ARC/INFO revision 6.0.1. Once the areal extent of the view boxes are set to the proper size (which varies with every slide), the registration coverage (transportation data layer) can be overlaid on the image in the OVERLAY window using the third mouse button when the cursor is in either the IMAGE or COVERAGE window (fig. 2B). If the image is not overlaid such that links, lines drawn between locations seen both in the image and in the registration coverage, can easily be generated, the view boxes in the COVERAGE and the IMAGE window can be resized and the image redrawn in the OVERLAY window using the described mouse button combination. Once a satisfactory overlay is achieved, the user may begin to establish links in the OVERLAY window. Links are established in the following order, from the image to the cover. Generally, links are established in the center of the image first, then working gradually towards the edge of the image in a circular manner (fig. 2B).

Links are established by placing the cursor in the OVERLAY window on a feature visible in the raster data, pressing and releasing mouse button one; in the same window move the cursor to the same feature visible in the vector data (coverage), press and release mouse button one again. A colored line (link) should appear between the two features (fig. 2B). When establishing links, select features that have locations which are stable over time such as road intersections, intersections of roads and streams, and dams. Features such as intermittent streams, islands within rivers, and lakes can have transient boundaries. Thus, these features may have changed from the time the USGS quadrangle maps were created and the time the CFSA aerial photography was obtained, causing errors in the image registration. Once four links are established, one in each of the four quadrants of the image, the image is registered by using the third mouse button with the cursor in the
OVERLAY window. Once complete, two additional windows titled "REGISTRATION ACTIONS" and "REGISTRATION RESULTS" appear on the screen. Click the DONE box; the REGISTRATION ACTIONS and REGISTRATION RESULTS windows disappear. Next, click the LOCK IMAGE button in the LINK ACTIONS window to make the program display the image based on the current set of links. Continue to establish links in the OVERLAY window. In a cyclic manner, add several links at a time, then using mouse button 3, reregister the image. The pop-up REGISTRATION ACTIONS window and REGISTRATION RESULTS window that appear each time the image is registered are used to continuously check the registration. Delete links with exceptionally long lengths by typing in the number of the link in the delete box within the REGISTRATION ACTIONS window. The RMS error should range from approximately 0 meters (perfect) to 5 meters (less desirable). The user should endeavor to keep RMS errors to a minimum; this study generally obtained RMS errors under 1.75 . This process requires that the larger-scaled images are registered to smaller-scaled coverages and large RMS errors could occur. The REGISTRATION RESULTS window, which appears each time the image is registered, displays the RMS error followed by a description of individual links, including the length of each link. More terrain relief in the representative image will require links of increased lengths resulting in a larger RMS error. The more terrain relief an image contains, the more difficult it is to register because the aerial photography is not ortho-rectified. It is impossible to identify an appropriate RMS for any particular image. The altitude and attitude of the aircraft differ from image to image as does the terrain relief. These factors all play important roles in the final RMS. This study used both the RMS from the registration and the visual fit of the image to the coverage to decide when a sufficient number of links had been established. Once the user determines that the best fit has been reached, the SAVE REGISTRATION button in the LINK ACTIONS window is clicked. When the registration for a particular image is saved, a file named $<\mathrm{tiff}>\mathrm{W}$ is created by ARC/INFO. This does not affect the TIFF file; the $<$ tiff $>$.W file is an additional file that contains the data needed by ARC/INFO to display and/or rectify the image. The ARC command RECTIFY must be used to combine the image and the tiff.W file into a new image which is stored in geographically referenced coordinates. After rectification, the image is converted to a 
A

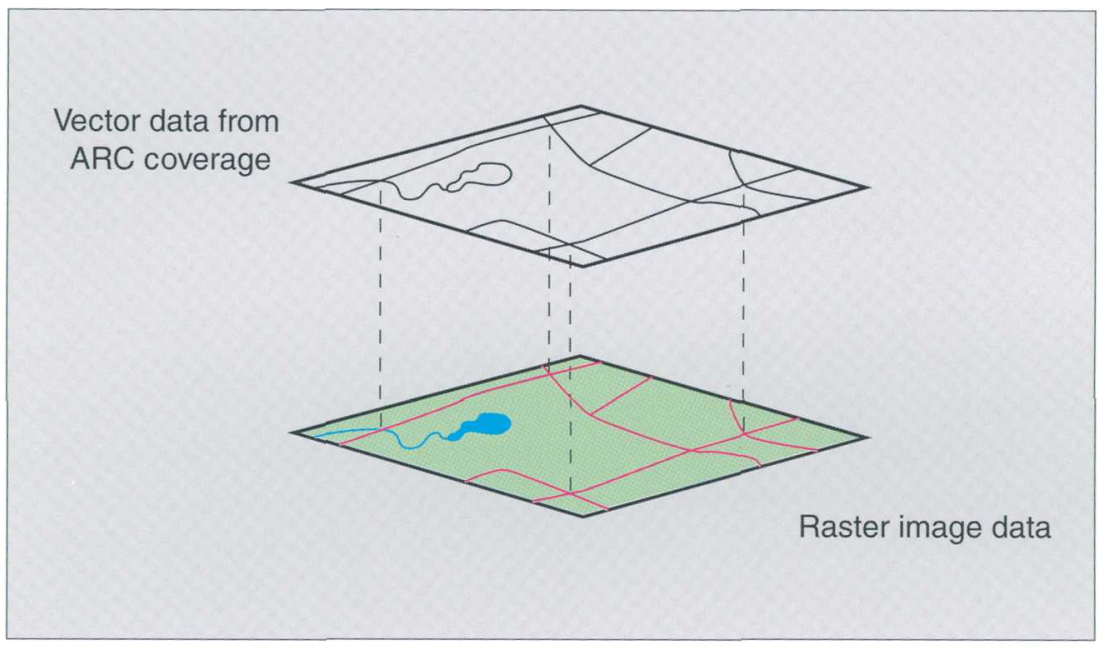

B
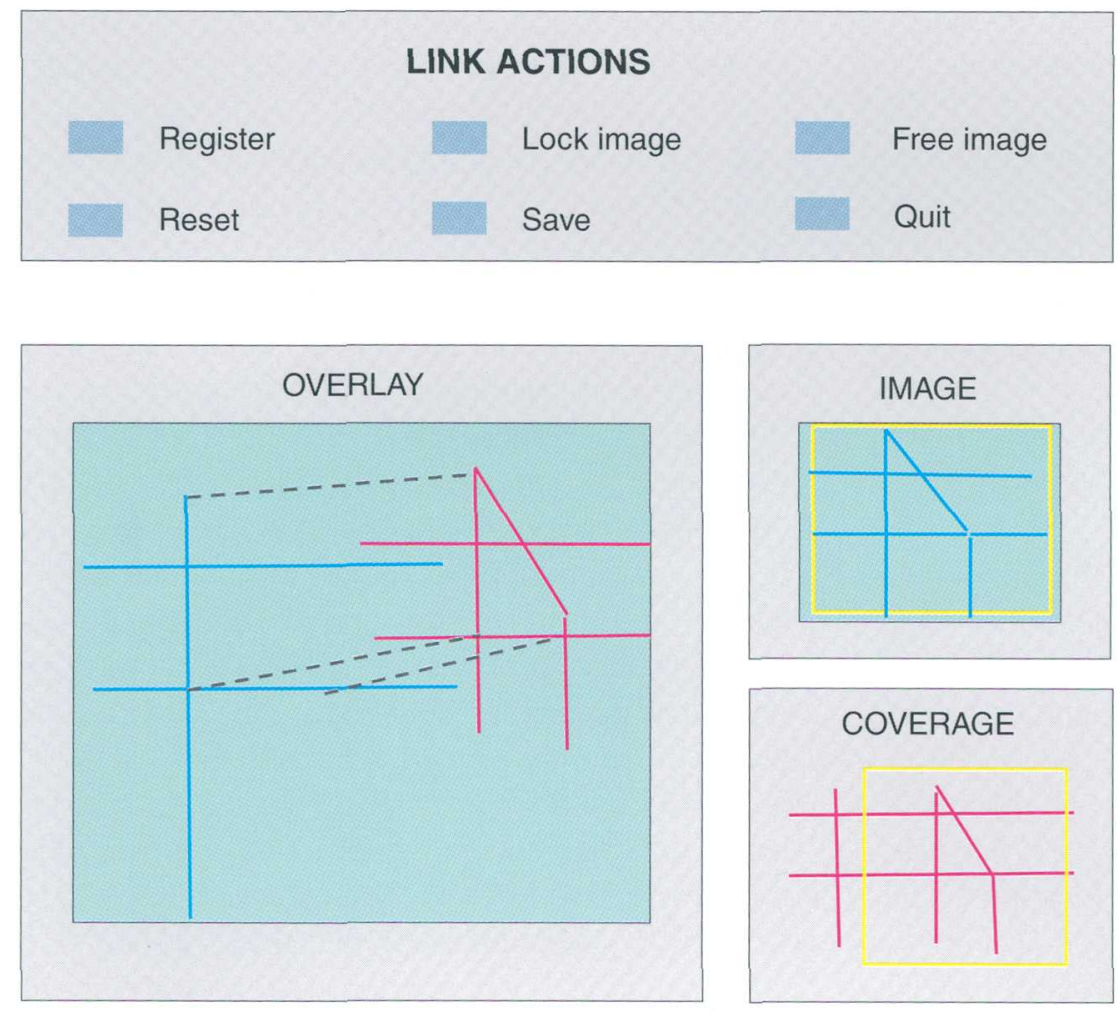

\section{EXPLANATION}

Roads

Surface water

Links established from image data to ARC coverage

\section{EXPLANATION}

Raster image data

Vector data from ARC coverage

Raster data within image data

Links established from image data to $A R C$ coverage

View box

Figure 2. A Diagrams showing an example of using a GIS data layer to graphically register a raster image. $\boldsymbol{B}$ Diagram showing an example of registration interface. 
multiband stacked grid (ARC/INFO raster data set) using the IMAGEGRID command in ARC. The grid stack can be displayed either in ARCPLOT, GRID, or ARCEDIT using the GRIDCOMPOSITE command.

\section{MERGING IMAGES TO ENCOMPASS AERIAL EXTENT OF STUDY AREA}

Commonly, multiple slide images must be merged to cover a selected area. To encompass the 2kilometer-radius spatial buffer area surrounding selected wells in this study, between 3 and 12 slides were required. The ARC grids (one per slide) were merged into a single grid (fig. 3) using the ARC/GRID module. To ensure that the same process was used for each site, cprocess.aml was developed to perform most of the processing steps. To allow the AML flexibility for multiple site processing, the AML was written to use images and grids which conform to an alphabetically prefixed naming convention, with ".tiff" as the suffix. For example, a group of six images would be named: a.tiff, b.tiff, c.tiff, d.tiff, e.tiff, and f.tiff. These images, once converted to grids by process.aml, had the suffix ".tiff” replaced by “_grd", such as "a_grd". This naming convention allowed process.aml to be used with very little manual editing. The users registered and rectified the images and rated the quality of the registration and the image with respect to other images for that site. This information was used to determine the order of precedence used by the GRID module during the merging process. When merging, the GRID module reads the precedence order set on the command line by the user. The output grid is written one cell at a time. The output cell value is set equal to the first NODATA value read from the input grids in order of precedence. Once a non-NODATA value is written for a cell, subsequent values for that cell from the input grids are ignored and the next cell is processed. Cprocess.aml was developed to rectify all images for a specific site, convert the rectified images to grids, then merge the grids into a composite grid based on the quality of the registration and the quality of the image. Cprocess.aml is described in appendix A and listed in appendix B. A larger project may require that several people register images. Some form of quality assurance/quality control (QAQC) should be maintained for the registration/rectification procedure. This study used separate people to register and rectify the images and to merge the grids. The information required by each step was transferred between person- nel via the use of a merge request form. In this manner, each individual served as the QAQC for processing performed during the previous step.

\section{VECTORIZATION OF DIGITAL IMAGE DATA}

The composite of the multiband stacked grids (created from the slide images), was used as the basis for delineating different land uses and crop types. Vectorization of image data (the conversion of image cells to line segments) was done manually. On-screen digitizing techniques were used to delineate field boundaries, urbanized areas, and rural built up areas. Several algorithms were developed in an effort to automate these processes.

\section{Manual Method of Crop Delineation}

The process of crop delineation is started by beginning an ARC session. The buffered roads are used as a template of the crop boundaries, thus the first step is to copy the buffered-roads coverage (described in the Transportation Data Layer section) into a coverage named "fields". Crop delineations (fig. 4) are generated using on-screen digitizing techniques in ARC/EDIT. The ARC/EDIT commands used were incorporated into setup.aml (appendix B). Setup.aml was used to ensure uniformity in the editing process. Setup.aml sets the initial editing environment and draws (in the background) the composite of the multiband grid stack. The crop data layer (fields coverage) initially consists of the buffered roads and is drawn as the edit coverage. The zoom functions in ARC/EDIT were used extensively to ensure that the accuracy of the crop boundaries was not compromised during the on-screen digitization process. Often, multiple zooms were used on the same area to achieve a displayed image where the user could easily see the pixel resolution.

The new crop data layer was saved and topology was built creating a polygon coverage using the ARC command BUILD. The CREATELABELS command was used to add a label point to each polygon in the coverage. These label points were subsequently used to attribute information specific to each polygon in the data layer. 

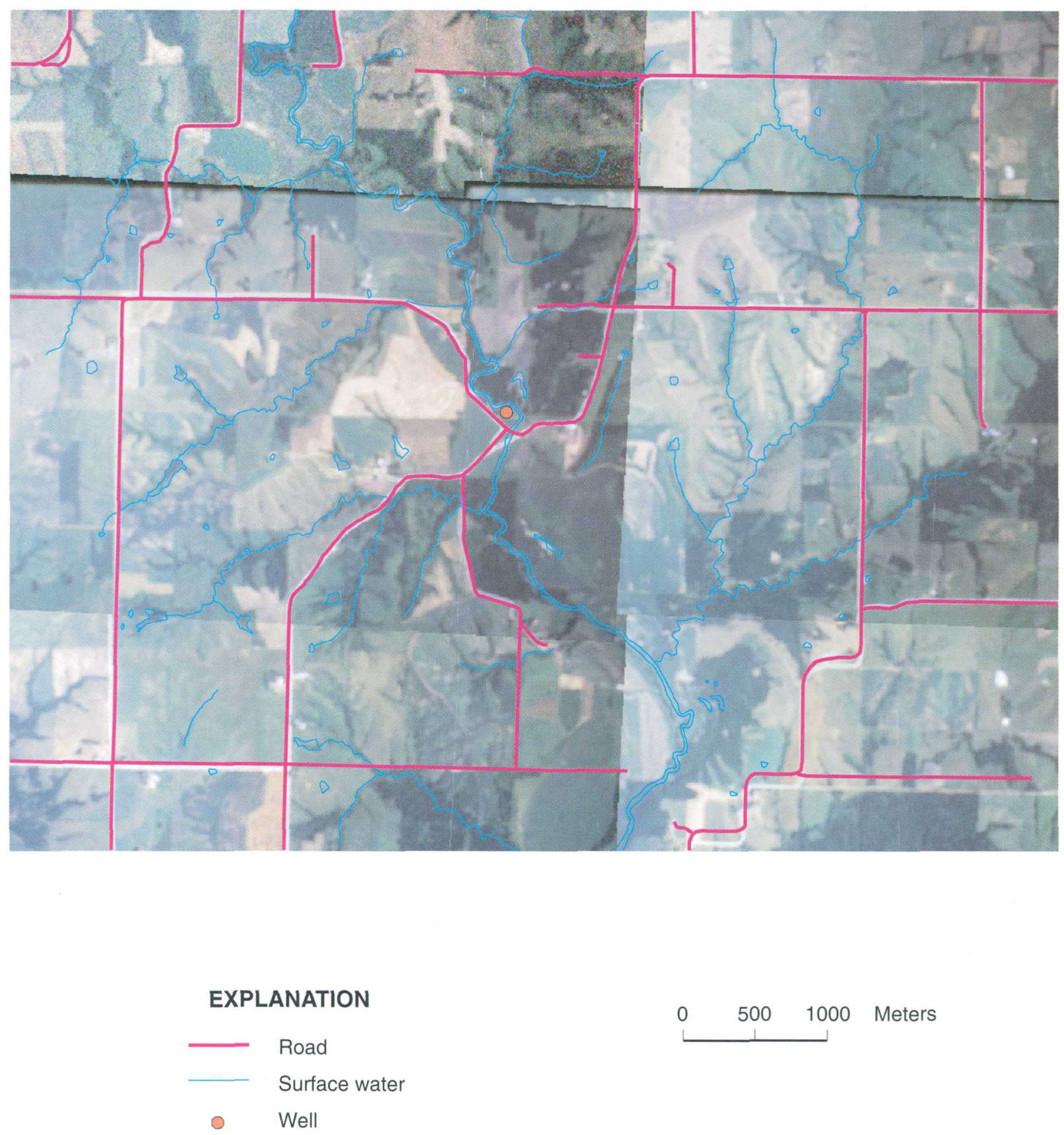

Figure 3. Example of transportation and hydrography data layers overlaid on a registered and rectified composite color image. 


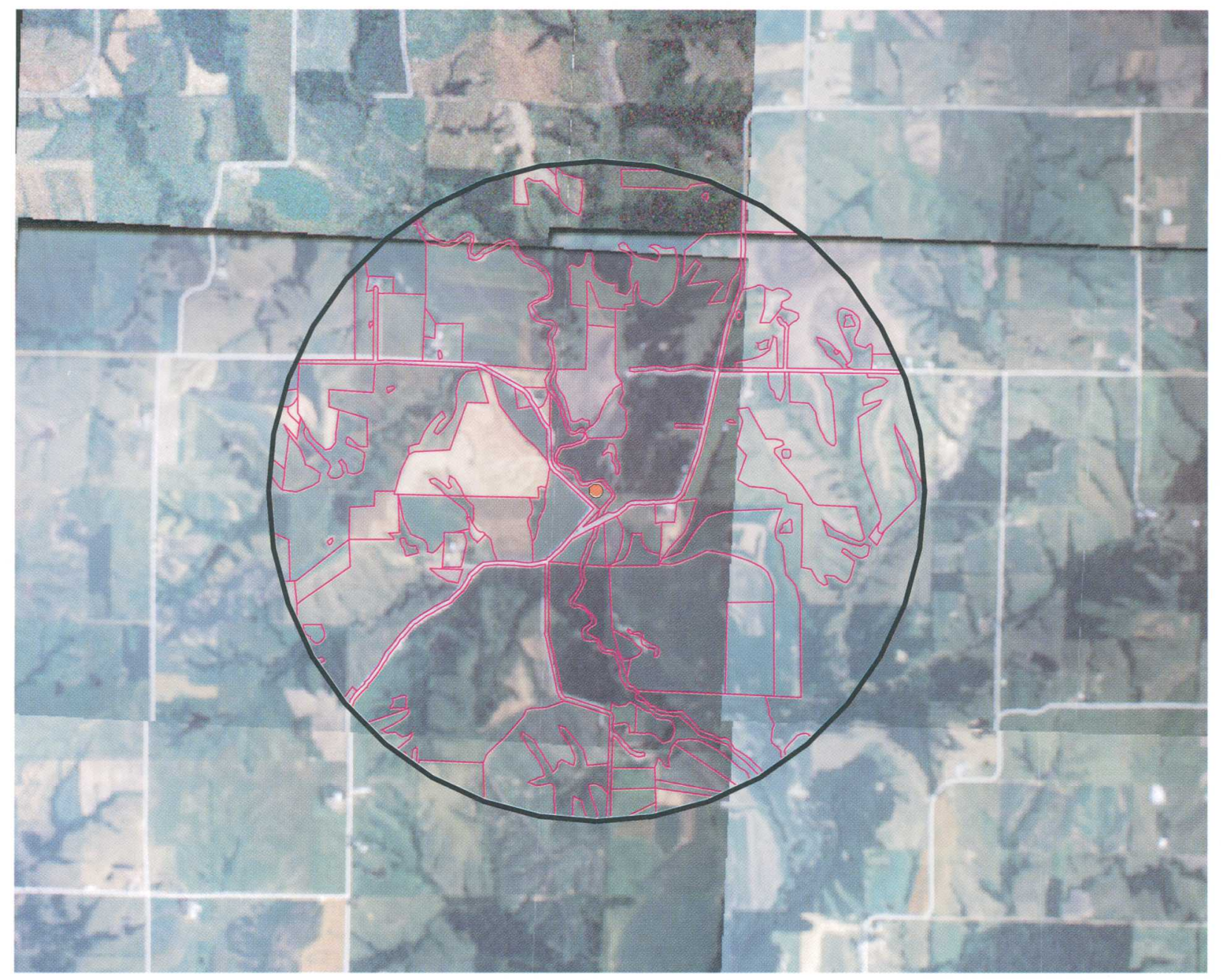

\section{EXPLANATION}

_ Spatial buffer (2 kilometer radius)

Field boundary

- Well

Figure 4. Example of field delineations overlaid on a registered and rectified composite color image. 


\section{Automated Attempts at Crop Delineation}

Several attempts were made to develop a pattern recognition process within the ARC/GRID module of ARC. The automated delineation attempts were not considered successful using this particular data source and are included in this report only to document the processes attempted.

The cell values obtained by scanning into TIFF image format are reflectance values of the visible light spectrum. An initial attempt grouped pixels by a range of reflective values representing colors, vectorized that data, then cleaned up the boundaries. However, because the difference in reflectance values was minimal, insufficient polygons were created. When the ranges of reflected values were narrowed, many polygons were created, few of which were actual crop boundaries.

A second attempt was made to delineate roads based on the cell value in the image instead of using the buffered polygon described above. This process, had it been successful, would have eliminated the need to buffer the roads providing for a more accurate determination of total area classified as roads. Initially, cells in the image with values within a specific range were selected and used to create a new grid. Each cell in the new grid was subsequently expanded by 2 cells. These new cells were filtered in such a manner that groups having less than or equal to 3 cells were deleted. This method removed single cells and smoothed edges of the remaining cell groups. However, the cleanup required through this process was so time consuming that it was more efficient to digitize the roads and create buffer zones around them.

Both of these attempts failed because of the image format used by this study. When using aerial photography from this source, reflectance values are too diverse to classify in an automated procedure. These photographs were never intended to be used for remote sensing, thus the controls necessary to use photography for this purpose were not in place. A field which is planted in soybeans might have many thousands of cells, each of which may have a unique reflectance value depending on many factors such as soil type, soil moisture, health of vegetation, sun angle, time of year, and time of day. These reflective values are not necessarily in any particular order. Therefore, a field may have as many unique colors as there are reflectance values. The images would need to be scanned into a Red, Green, Blue (RGB) image format for a viable automated procedure to be developed.
DATA CLASSIFICATION PROCEDURE

The manual method was based on on-screen digitization to classify data according to information obtained from the CFSA crop maps. Several attempts were made to develop automated procedures to classify land use. The automated procedures were based on reflective values which were attributes of the raster data to develop classification schemes. Due to source-data and time limitations, the manual method proved to be the most efficient alternative for this study.

\section{Manual Method of Data Classification}

The USDA CFSA county offices maintain annual crop maps. The attributes for these maps are obtained from the farmers that participate in USDA farm programs. The manual method of data classification was developed using CFSA crop maps, ARC/ EDIT, and ARC/INFO's image integrator. Several AML programs were developed to assist in the editing procedure (setup.aml, both.aml, none.aml, colordraw.aml) (appendixes A and B). These programs perform various repetitive tasks, such as setting up the drawing environment and querying the user to select and attribute polygon labels. The user periodically saves the coverage, upon which the coverage is redrawn, and the attributed polygons are filled using a color scheme that is predefined in the AML program. A visual comparison is made of the digital map displayed on the computer screen with the hard-copy CFSA crop maps. Using these CFSA crop maps as source data, the fields of a specific crop type (for example, corn) are selected, then the INFO item TYPE is calculated equal to the value that represents that crop $($ corn $=70)$. The coverage is then saved and upon redraw, the corn fields are shaded with a predefined color. This method allows for easy identification of fields which are not yet attributed with a crop type. The shaded fields (fig. 5) also make spatial location of fields on the hard-copy CFSA crop maps more efficient. The fields not defined on the CFSA crop maps (fig. 5) are then classified using surrounding fields in the area as a 'visual' training set and a classification determination is made on the basis of the image alone. This classification method provides land use for the entire 2-kilometer-radius spatial buffer area surrounding a selected well (fig. 6). 


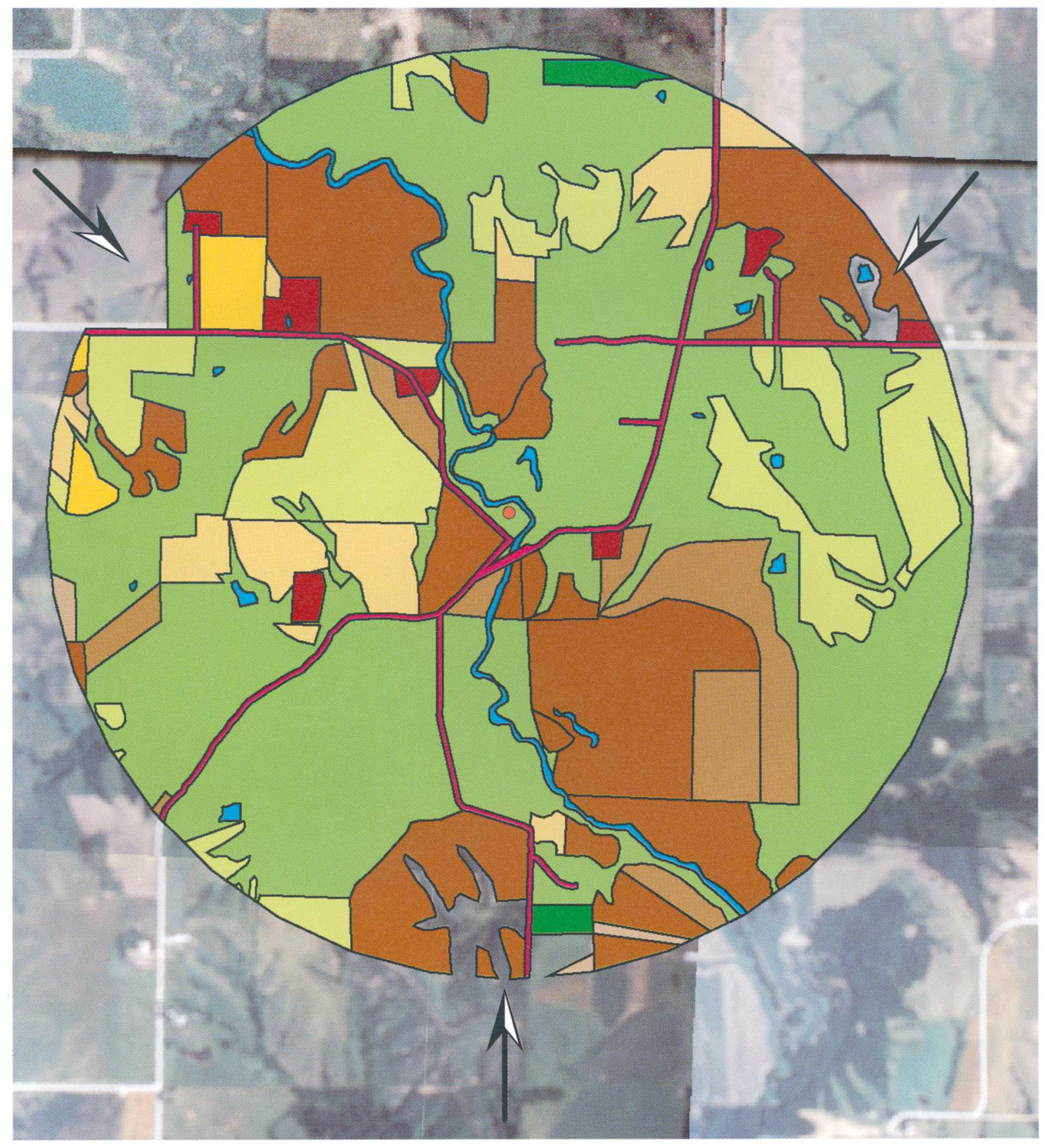

\section{EXPLANATION}

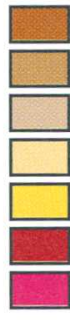

Corn

Soybeans

Oats

Hay

Pasture

Rural build up

Road
Land in Conservation

Reserve Program

Mixed forest

Grass

Surface water

- Well

Arrows indicate gaps

in CFSA data

Figure 5. Examples of data classified using Consolidated Farm Services Agency (CFSA) crop maps and unclassified areas representing undocumented classifications in the CFSA crop data. 


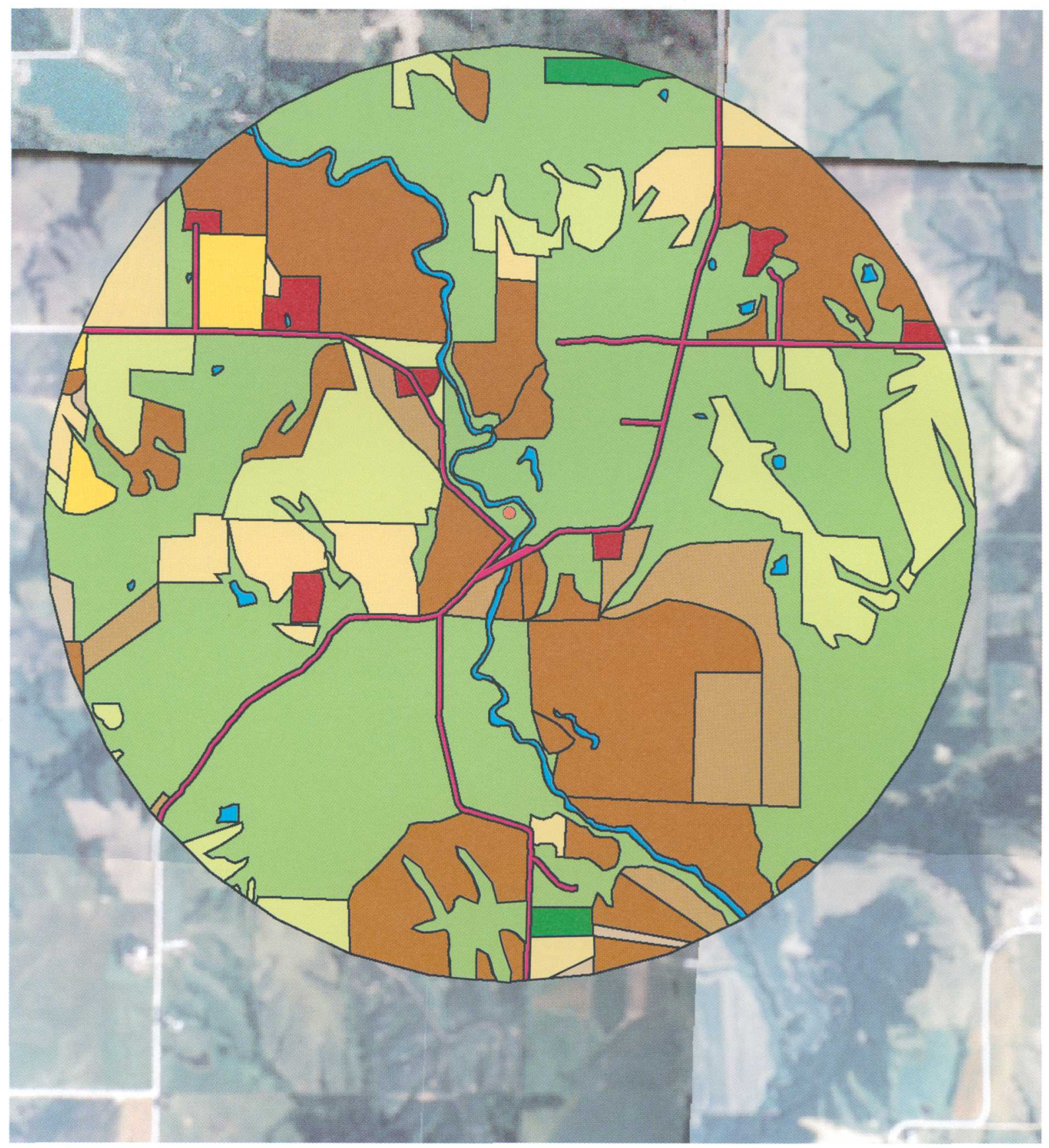

\section{EXPLANATION}

\begin{tabular}{l}
$\square$ Corn \\
\hline $\begin{array}{l}\text { Soybeans } \\
\text { Oats }\end{array}$ \\
Hay \\
\hline$\square$ Pasture \\
Rural build up \\
Road
\end{tabular}

Land in Conservation Reserve Program

0 Mixed forest

Grass

Surface water

- Well

Figure 6. Example of data classification completed using Consolidated Farm Services Agency (CFSA) crop maps and classification using areas with documented classification as visual training areas. 


\section{Automated Attempts at Data Classification}

Several methods were attempted to automate data classification, none of which were successful. One procedure attempted to classify the crops after the fields had been manually delineated. The delineated crop coverage is converted to a grid data layer (raster data). The cell values of the grid data layer, obtained from the scanned image TIFF file represented the reflectance values of the visible light spectrum. These two data layers are merged such that the new grid data layer has a single value for each field delineated, this value being the mean of all values of the cells comprising that field. An automated classification scheme using this method was not successful because more than a $50 \%$ overlap of the classifications occurred on each end of the scale. Furthermore, a classification scheme had to be created for each image using this method. The altitude, attitude, flight direction of the aircraft, the time day, and the time of year the photograph was taken varied such that the range of values representing any particular crop type was so dissimilar that this method of automated classification was not possible using TIFF images created from this type of photography.

A second method attempted to use single-cell classification. Numerous single-cell classification schemes were developed which attempted to provide consistent, accurate classification of the data. However, for similar reasons described above, this second attempt also proved unsuccessful.

It is hypothesized that if the photos were scanned in RGB, a classification scheme could be developed which would allow for some measure of automated classification. However, it is felt that the accuracy of a possible automated classification procedure still would be inferior to that provided by the CFSA crop maps.

\section{SUGGESTIONS FOR METHOD IMPROVE- MENT}

The following suggestions are made after having registered and classified approximately 1500 images for this study.

- A classification could be added to the data set to keep track of how the classification was made, for example, $0=$ CFSA crop map, $1=$ visual interpretation.

- An attribute could be added to the transportation data to differentiate road types, such as gravel roads, county highways, state highways, and interstates. This attribute could be used to selectively buffer the roads by different widths based on the road type, wider for interstates, narrower for gravel roads. This approach could improve the accuracy of the areal measurement of the roads.

- The images could be scanned in RGB format. The automated procedures described above may be adapted to work with multiple-banded data.

\section{SUMMARY}

Land-use data were compiled using GIS methods developed within ARC/INFO to integrate aerial photographs, digitized data, and data from CFSA crop maps. Aerial photography consisting of 35-mm slides were scanned into TIFF files, registered into a geographically referenced coordinate system, rectified, and integrated into GIS data sets. Boundaries between land use were delineated from these GIS data sets using on-screen digitizing techniques. Crop types were determined using information obtained from U.S. Department of Agriculture, CFSA offices. Crop information not supplied by the CFSA were attributed by manual classification procedures. AML programs written for this study were used to automate repetitive or time consuming steps and to provide consistency within the data set. Automated methods were tested to provide delineation of the fields and classification of land use, but proved less efficient and accurate than manual methods. 


\section{REFERENCES}

Adobe Systems Incorporated, 1993, Adobe Photoshop users guide, version 2.1 for Macintosh: Mountain View, Calif. p. 5-7.

Environmental Systems Research Institute, Inc. (ESRI), 1994, ARC/INFO users guide, version 7.0: Redlands, Calif., 12 p.

Cartier, K. D., Peltz, L. A., and Smith, J. LaRue, 1993, Development and documentation of spatial data bases for the Lake Tahoe basin, California and Nevada: U.S. Geological Survey Water-Resources Investigations Report 93-4182, 65 p.

Kolpin, D.W., and Burkart, M.R., 1991, Work plan for regional reconnaissance for selected herbicides and nitrate in near-surface aquifers of the Midcontinental United States: U.S. Geological Survey Open-File Report 91-59, 18 p.

Kolpin, D.W., Burkart, M.R., and Thurman, E.M., 1993, Hydrogeologic, water-quality, and land-use data for the reconnaissance for herbicides and nitrate in nearsurface aquifers of the Midcontinental United States, 1991: U.S. Geological Survey Open-File Report 93-114, $61 \mathrm{p}$.

Kolpin, D.W., Burkart, M.R., and Thurman, E.M., 1994, Herbicides and nitrate in near-surface aquifers in the Midcontinental United States, 1991: U.S. Geological Survey Water-Supply Paper 2413, 34 p.

Kolpin, D.W., Goolsby, D.A., and Thurman, E.M., 1995, Pesticides in near-surface aquifers: An assessment using highly sensitive analytical methods and tritium: Journal Environmental Quality v. 24, p. 1,125-1,132.

Kolpin, D.W., and Thurman, E.M., 1995, Postflood occurrence of selected agricultural chemicals and volatile organic compounds in near-surface unconsolidated aquifers in the upper Mississippi River Basin, 1993: U.S. Geological Survey Circular 1120-G, 20 p.

Nebert, D.D., 1989, Review of edgematching procedures for digital cartographic data used in geographic information systems (GIS): U.S. Geological Survey Open-File Report 89-579, p. 2. 
APPENDIXES 


\section{APPENDIX A}

Description of AMLs included in Appendix B.

\begin{abstract}
AML NAME
Setup.aml

Aeshades.aml

Colordraw.aml

Both.aml

\section{BRIEF DESCRIPTION}

Establishes edit environment for corrections to the vector data and data classification. The four options are listed below.

ARCEDIT

An option to setup.aml which shades the fields with the designated color as they are classified. The image is not drawn when this option is used.

ARCEDIT

An option to setup.aml which draws the registered/rectified color composite images in the background of the ARCEDIT draw environment. The classified data are not shaded when this option is used

ARCEDIT

An option to setup.aml which draws the registered/rectified composite color images in the background and shades the fields with the designated color as they are classified. This is the slowest of the four options to setup.aml.
\end{abstract}

None.aml

Cprocess.aml

ARC/GRID
ARCEDIT An option to setup.aml which features only the vector data in the ARCEDIT draw environment. It is the fastest option to setup.aml.

Rectifies the registered images, converts the images to grids, merges the grids into a single grid, processes cells with zero values to NODATA, creates multiple buffers around the well, buffers the road coverage and creates the field coverages, adds attribute items to the field coverage, appends the hydrology to the field coverage, and plots the composite of the multi-band stacked grids for visual inspection. Two lines at the top of this aml are edited before use at each well site. This aml can take several hours to complete.

Dig.aml ARCEDIT Sets up the editing environment for use during the heads-up digitizing of the field/crop boundaries. 


\section{APPENDIX B}

Listing of AML programs developed for use with the land-use classification phase of the study. By setting the \&AMLPATH to the directory where the AMLs are located, the AMLS can be initiated from any directory.

\section{Setup.aml}

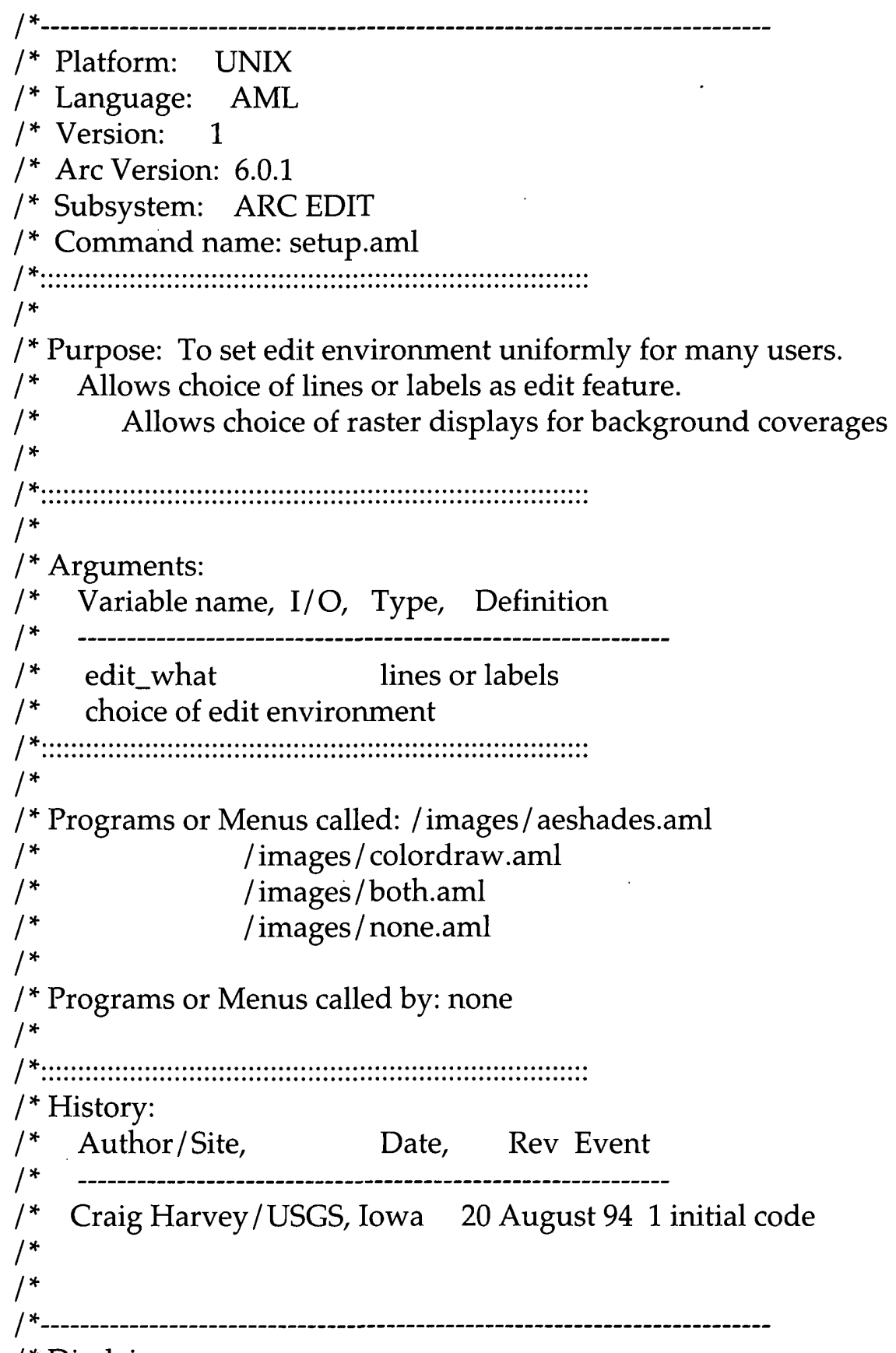




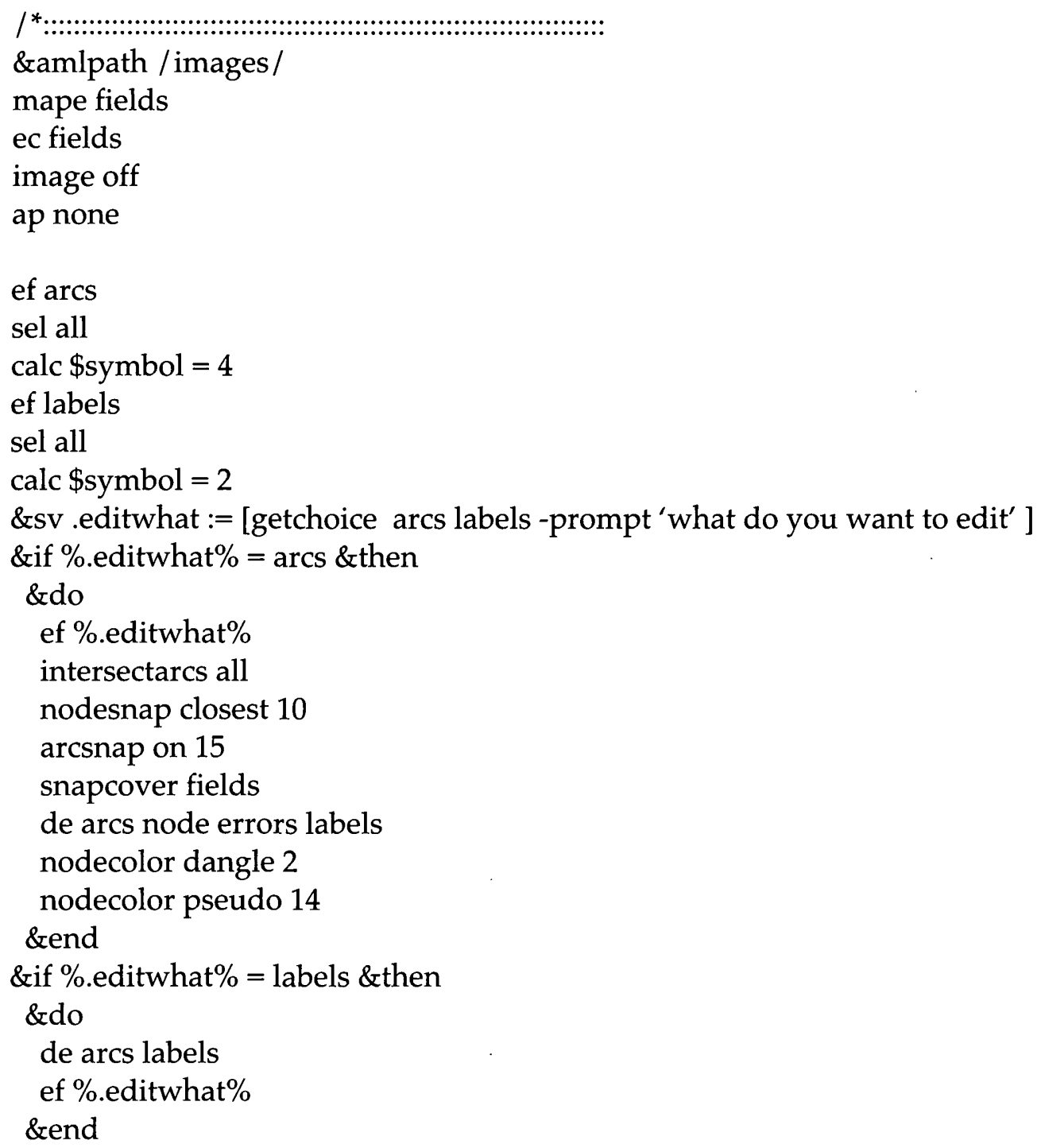

\&sv .choice $:=$ [getchoice ../ aeshades.aml ../ colordraw.aml both none -prompt 'Select the ArcPlot or IMAGE display option for this ArcEdit session']

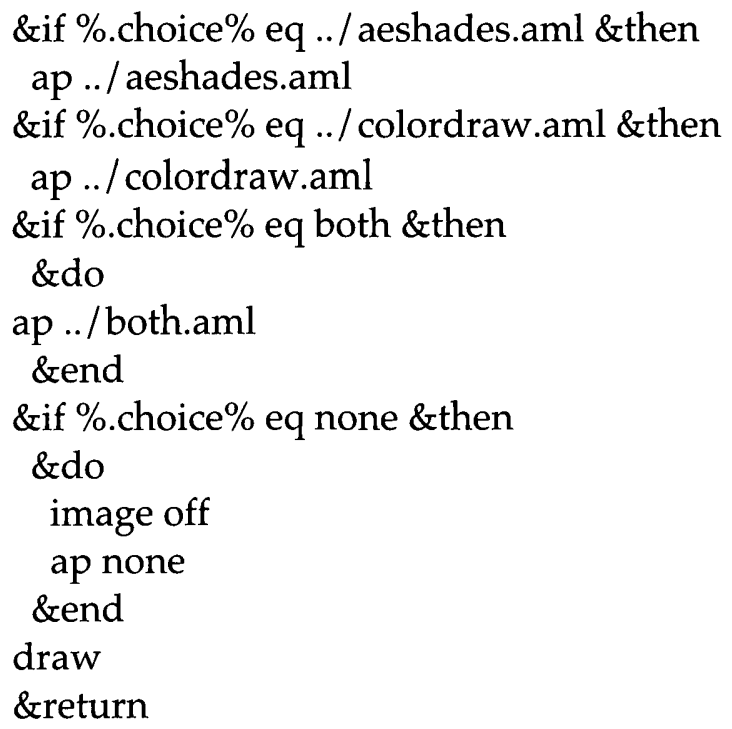




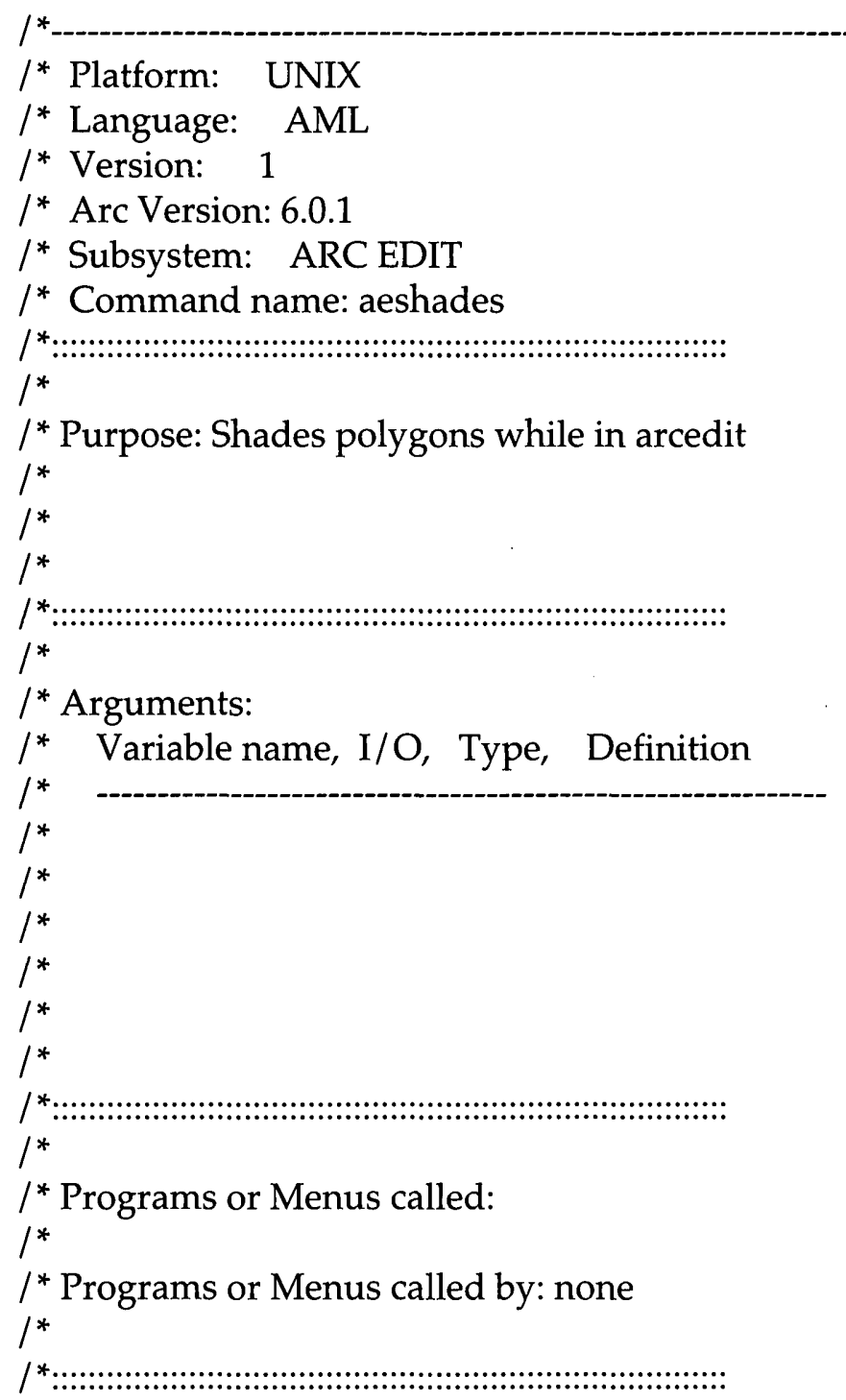

I* History:

I* Author/Site, Date, Rev Event

I*

I* Craig Harvey/USGS, Iowa 20 August 941 initial code

$1^{*}$

$1 *$

$1^{*}$

I* Disclaimer:

I*Although this program had been used by the U. S. Geological Survey, I*no warranty, expressed or implied, is made by the USGS as to the accuracy $1 *$ and fuctionality of the program and related program material nor shall the fact $1 *$ of distribution constitute any such warranty, and no responsibility is assumed / *by the USGS in connection herewith.

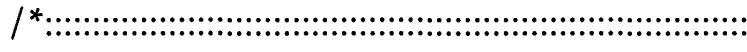

shadeset colornames

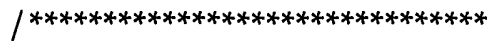


$l^{*}$ BUILT-UP AREA

/*select urban

reselect fields poly class $=10$

shadesymbol 125

shadeput 510

polygonshades fields 1000

clearselect

/*select rural urban

reselect fields poly class $=11$

shadesymbol 123

shadeput 511

polygonshades fields 1000

clearselect

$/{ }^{*}$ select golf course

reselect fields poly class $=12$

shadesymbol 71

shadeput 512

polygonshades fields 1000

clearselect

$/{ }^{*}$ select roads

reselect fields poly class $=13$

shadeset colornames

shadesymbol 13

shadeput 513

polygonshades fields 1000

clearselect

/*select airports

reselect fields poly class $=14$

shadeset colornames

shadesymbol 11

shadeput 514

polygonshades fields 1000

clearselect

${ }^{*}$ select feedlots

reselect fields poly class $=15$

shadeset colornames

shadesymbol 100

shadeput 515

polygonshades fields 1000

clearselect

/ *select railroad switch yard

reselect fields poly class $=16$

shadeset colornames

shadesymbol 117

shadeput 516 
polygonshades fields 1000

clearselect

/*select waste disposal sites

reselect fields poly class $=17$

shadeset colornames

shadesymbol 112

shadeput 517

polygonshades fields 1000

clearselect

/********************************

/* TREE COVÉR

I*select trees - forested

reselect fields poly class $=20$

shadesymbol 76

shadeput 520

polygonshades fields 1000

clearselect

$/{ }^{*}$ select mixed forest

reselect fields poly class $=21$

shadesymbol 72

shadeput 521

polygonshades fields 1000

clearselect

/********************************

$I^{*}$ WATER

/ *select rivers + streams

reselect fields poly class $=30$

shadesymbol 53

shadeput 530

polygonshades fields 1000

clearselect

/ ${ }^{*}$ select Lakes and Ponds

reselect fields poly class $=31$

shadesymbol 55

shadeput 531

polygonshades fields 1000

clearselect

/*select wetlands/swamps

reselect fields poly class $=32$

shadesymbol 59

shadeput 532

polygonshades fields 1000

clearselect 
$1 * * * * * * * * * * * * * * * * * * * * * * * * * * * * * * * *$

/* CEREAL GRAINS

$/{ }^{*}$ select unknown cereal

reselect fields poly class $=40$

shadesymbol 92

shadeput 540

polygonshades fields 1000

clearselect

${ }^{*}$ select mixed oats

reselect fields poly class $=41$

shadesymbol 85

shadeput 541

polygonshades fields 1000

clearselect.

/*select wheat

reselect fields poly class $=42$

shadesymbol 95

shadeput 542

polygonshades fields 1000

clearselect

/*select Barley

reselect fields poly class $=43$

shadesymbol 96

shadeput 543

polygonshades fields 1000

clearselect

${ }^{*}$ select rye

reselect fields poly class $=44$

shadesymbol 89

shadeput 544

polygonshades fields 1000

clearselect

/ ${ }^{*}$ select milo

reselect fields poly class $=45$

shadesymbol 99

shadeput 545

polygonshades fields 1000

clearselect

/ ${ }^{*}$ select Triticale

reselect fields poly class $=46$

shadesymbol 98

shadeput 546

polygonshades fields 1000

clearselect 
/*select millet

reselect fields poly class $=47$

shadesymbol 101

shadeput 547

polygonshades fields 1000

clearselect

$1 * * * * * * * * * * * * * * * * * * * * * * * * * * * * * * *$

I* FORAGE AND/OR PROGRAM ACRES

${ }^{*}$ select hay (alfalfa, etc...) and forage and brome

reselect fields poly class $=51$

aselect fields poly class $=50$

aselect fields poly class $=52$

shadesymbol 71

shadeput 550

polygonshades fields 1000

clearselect

$/{ }^{*}$ select pasture

reselect fields poly class $=53$

shadesymbol 68

shadeput 553

polygonshades fields 1000

clearselect

/*select native grass

reselect fields poly class $=54$

shadesymbol 67

shadeput 554

polygonshades fields 1000

clearselect

/ *select grass

reselect fields poly class $=55$

aselect fields poly class $=59$

shadesymbol 75

shadeput 555

polygonshades fields 1000

clearselect

/*select acr / crp

reselect fields poly class $=56$

aselect fields poly class $=57$

aselect fields poly class $=58$

shadesymbol 73

shadeput 556

polygonshades fields 1000

clearselect 
/*****************************

$l^{*}$ other crops

/*select sugar beets

reselect fields poly class $=60$

shadesymbol 118

shadeput 560

polygonshades fields 1000

clearselect

/*select melons

reselect fields poly class $=61$

shadesymbol 72

shadeput 561

polygonshades fields 1000

clearselect

${ }^{*}$ select orchards

reselect fields poly class $=62$

shadesymbol 124

shadeput 562

polygonshades fields 1000

clearselect

/*select garden

reselect fields poly class $=63$

shadesymbol 112

shadeput 563

polygonshades fields 1000

clearselect

$1 * * * * * * * * * * * * * * * * * * * * * * * * * * * * *$

/* ROW CROPS

${ }^{*}$ select corn

reselect fields poly class $=70$

shadesymbol 84

shadeput 570

polygonshades fields 1000

clearselect

/ *select beans

reselect fields poly class $=71$

shadesymbol 92

shadeput 571

polygonshades fields 1000

clearselect

${ }^{*}$ select sorgum

reselect fields poly class $=72$

shadesymbol 103 
shadeput 572

polygonshades fields 1000

clearselect

/*select potatoes

shadeset colornames.shd

reselect fields poly class $=73$

shadesymbol 90

shadeput 573

polygonshades fields 1000

clearselect

/*select OTHER BEANS (pinto, kidney, green)

shadeset colornames.shd

reselect fields poly class $=74$

shadesymbol 62

shadeput 574

polygonshades fields 1000

clearselect

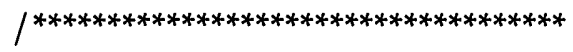

$I^{*}$ usage class

$/{ }^{*}$ select non ag

reselect fields poly class $=98$

shadesymbol 107

shadeput 598

polygonshades fields 1000

clearselect

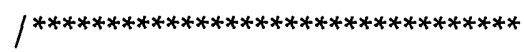

/* TILLAGE PRACTICE, ETC.

shadeset plotter.shd

$I^{*}$ double cropped

reselect fields poly double $=1$

shadesymbol 9

shadeput 600

polygonshades fields 1000

clearselect

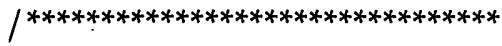

$I^{*}$ irrigated

reselect fields poly irrig $=1$

shadecolor 13

shadesymbol 21

shadeput 601

polygonshades fields 1000

clearselect 
$/ *$ till

${ }^{*}$ till $-=0$, conventional tillage

${ }^{*}$ till $=1$, notill

$l^{*}$ till $=2$, high residue

$l^{*}$ till $=3$, medium residue

$l^{*}$ till $=4$, low residue

reselect fields poly till eq 1 shadesymbol 81

shadecolor 5

/ *shadeput 602

polygonshades fields 1000

clearselect

reselect fields poly till eq 2

shadesymbol 77

shadecolor 4

/*shadeput 602

polygonshades fields 1000

clearselect

reselect fields poly till eq 3 shadesymbol 65

shadecolor 6

/*shadeput 602

polygonshades fields 1000

clearselect

reselect fields poly till eq 4 shadesymbol 97

shadecolor 8

/ shadeput 602

polygonshades fields 1000

clearselect

$1 * * * * * * * * * * * * * * * * * * * * * * * * * * * * * * * * * *$

$I^{*}$ cultivation practice

${ }^{*}$ cult $=0$, conventional

$/{ }^{*}$ cult $=1$, drilled

$/{ }^{*}$ cult $=2$, not intertilled between rows

reselect fields poly cult ne 0

shadesymbol 65

shadeput 603

polygonshades fields 1000

clearselect

/********************************

${ }^{*}$ failed crop

${ }^{*}$ fail $=0$, survived

$l^{*}$ fail $=1$, failed 
reselect fields poly fail $=1$

shadesymbol 65

shadeput 604

polygonshades fields 1000

clearselect

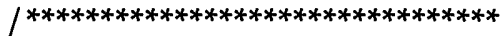

$I^{*}$ highly erodable

$l^{*}$ erode $=0$, non-highly erodible land ("NHEL" on CFSA crop maps)

$/$ *erode $=1$, highly erodible land ("HEL" on CFSA crop maps)

reselect fields poly erode $=1$

shadesymbol 57

shadecolor 2

shadeput 605

polygonshades fields 1000

clearselect

\&return

\section{Colordraw.aml}

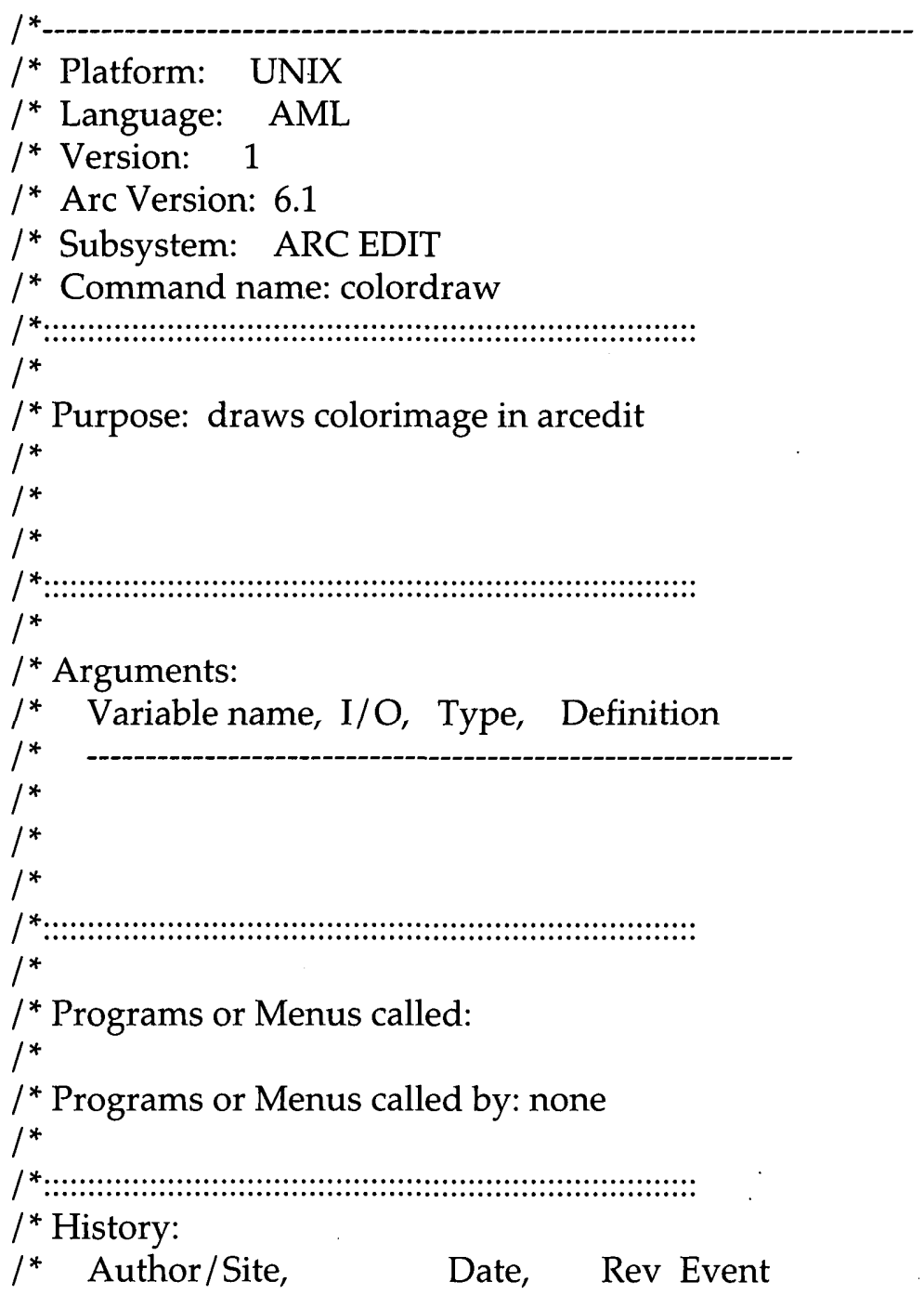




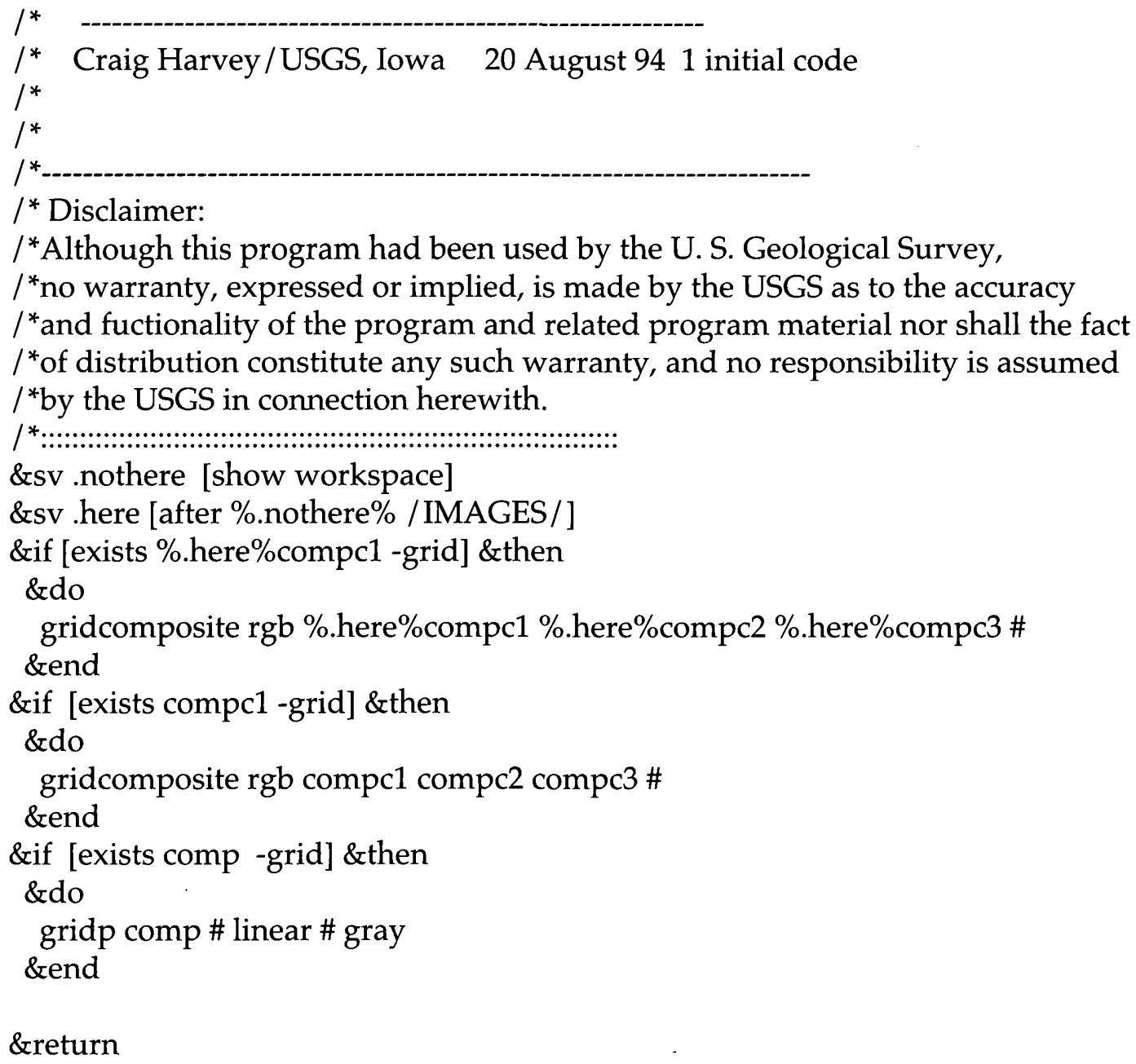

/ Although this program had been used by the U. S. Geological Survey, I*no warranty, expressed or implied, is made by the USGS as to the accuracy $I^{*}$ and fuctionality of the program and related program material nor shall the fact $/{ }^{*}$ of distribution constitute any such warranty, and no responsibility is assumed $/$ *by the USGS in connection herewith.

\&sv .nothere [show workspace]

\&sv .here [after \%.nothere\% / IMAGES/]

\&if [exists \%.here\%compc1 -grid] \&then

\&do

gridcomposite rgb \%.here\%compc1 \%.here\%compc2 \%.here\%compc3 \# \&end

\&if [exists compc1 -grid] \&then

\&do

gridcomposite rgb compc1 compc2 compc3 \#

\&end

\&if [exists comp -grid] \&then

\&do

gridp comp \# linear \# gray

\&end

\&return

\section{Both.aml}

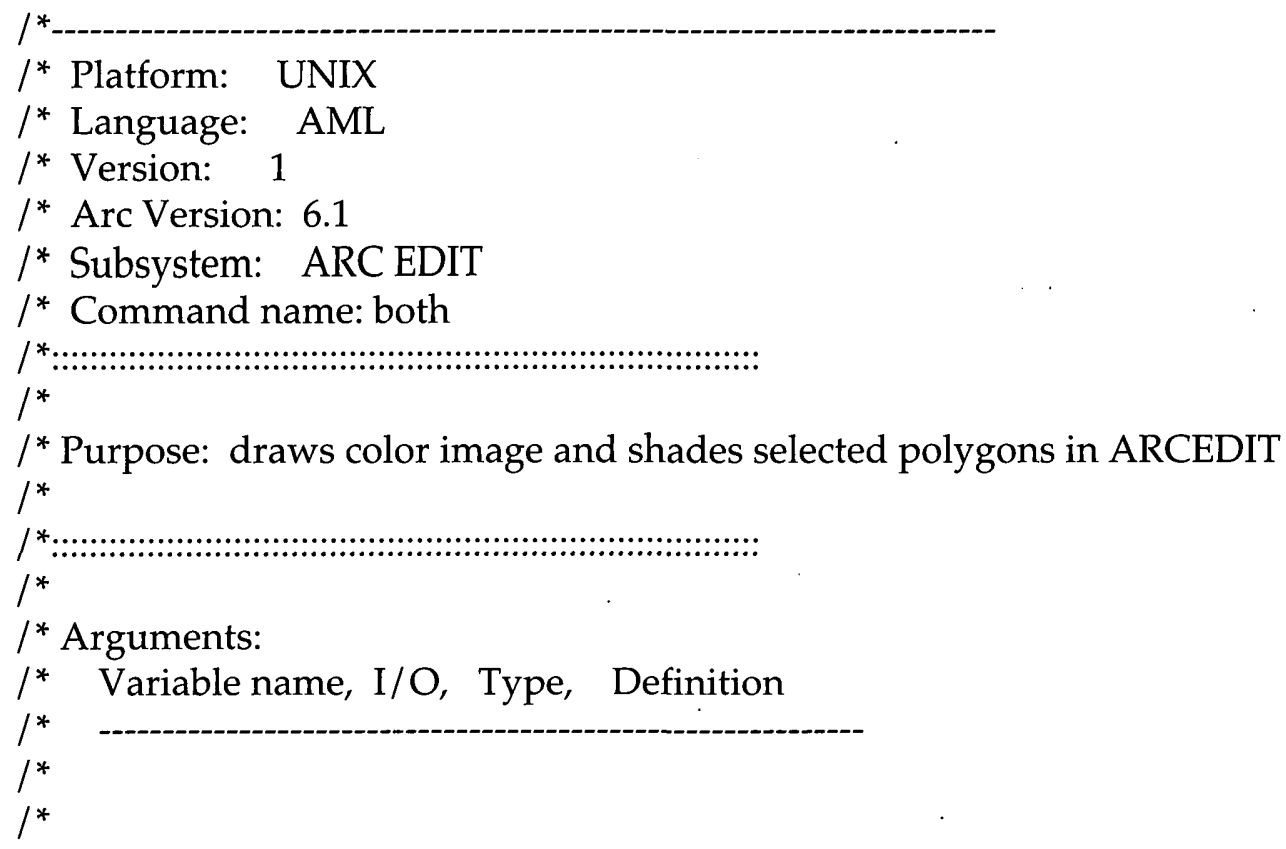




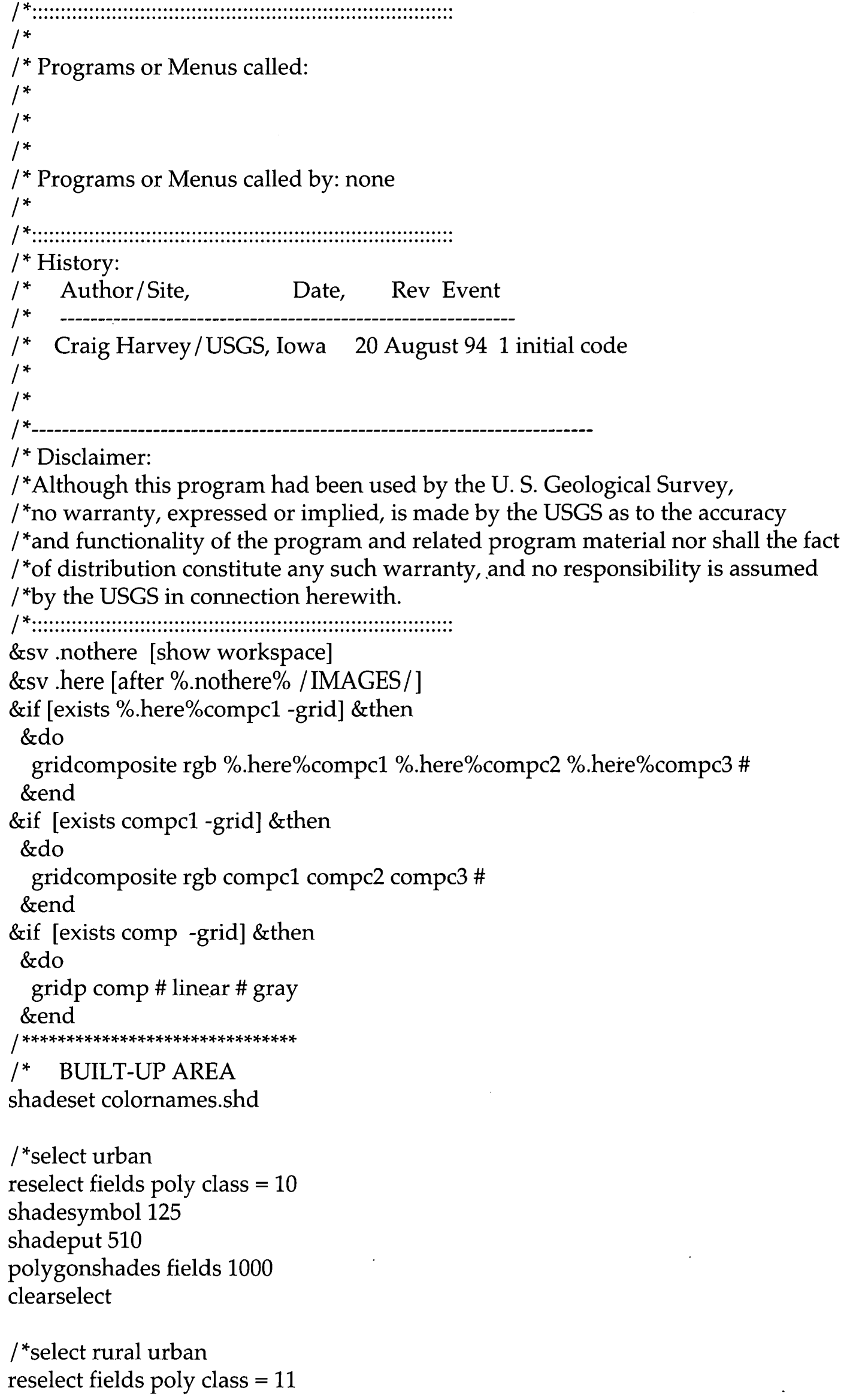

$1^{*}$ Although this program had been used by the U. S. Geological Survey, $/{ }^{*}$ no warranty, expressed or implied, is made by the USGS as to the accuracy $/^{*}$ and functionality of the program and related program material nor shall the fact $I^{*}$ of distribution constitute any such warranty, and no responsibility is assumed $/$ *by the USGS in connection herewith.

$I^{*}::$ :

\&sv .nothere [show workspace]

\&sv .here [after \%.nothere\% / IMAGES/]

\&if [exists \%.here\%compc1 -grid] \&then

\&do

gridcomposite rgb \%.here \%compc1 \%.here \%compc2 \%.here\%compc3 \# \&end

\&if [exists compc1 -grid] \&then

\&do

gridcomposite rgb compc1 compc2 compc3 \#

\&end

\&if [exists comp -grid] \&then

\&do

gridp comp \# linear \# gray \&end

/********************************

/* BUILT-UP AREA

shadeset colornames.shd

/*select urban

reselect fields poly class $=10$

shadesymbol 125

shadeput 510

polygonshades fields 1000

clearselect

/*select rural urban

reselect fields poly class $=11$ 
shadesymbol 123

shadeput 511

polygonshades fields 1000

clearselect

$/{ }^{*}$ select golf course

reselect fields poly class $=12$

shadesymbol 71

shadeput 512

polygonshades fields 1000

clearselect

$/{ }^{*}$ select roads

reselect fields poly class $=13$

shadeset colornames

shadesymbol 13

shadeput 513

polygonshades fields 1000

clearselect

/ ${ }^{*}$ select airports

reselect fields poly class $=14$

shadeset colornames

shadesymbol 11

shadeput 514

polygonshades fields 1000

clearselect

/ select feedlots

reselect fields poly class $=15$

shadeset colornames

shadesymbol 100

shadeput 515

polygonshades fields 1000

clearselect

$/{ }^{*}$ select railroad switch yard

reselect fields poly class $=16$

shadeset colornames

shadesymbol 117

shadeput 516

polygonshades fields 1000

clearselect

/ *select waste disposal sites

reselect fields poly class $=17$

shadeset colornames

shadesymbol 112

shadeput 517

polygonshades fields 1000

clearselect 
/********************************

/* TREE COVER

/*select trees - forested

reselect fields poly class $=20$

shadesymbol 76

shadeput 520

polygonshades fields 1000

clearselect

/*select mixed forest

reselect fields poly class $=21$

shadesymbol 72

shadeput 521

polygonshades fields 1000

clearselect

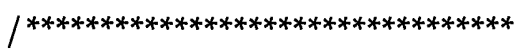

${ }^{*}$ WATER

$/{ }^{*}$ select rivers + streams

reselect fields poly class $=30$

shadesymbol 53

shadeput 530

polygonshades fields 1000

clearselect

/ select Lakes and Ponds

reselect fields poly class $=31$

shadesymbol 55

shadeput 531

polygonshades fields 1000

clearselect

/*select wetlands/swamps

reselect fields poly class $=32$

shadesymbol 59

shadeput 532

polygonshades fields 1000

clearselect

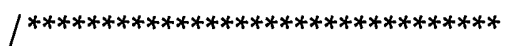

/* CEREAL GRAINS

/*select unknown cereal

reselect fields poly class $=40$

shadesymbol 92

shadeput 540

polygonshades fields 1000

clearselect

/*select mixed oats

reselect fields poly class $=41$

shadesymbol 85

shadeput 541

polygonshades fields 1000 
clearselect

I*select wheat

reselect fields poly class $=42$

shadesymbol 95

shadeput 542

polygonshades fields 1000

clearselect

\author{
I*select Barley \\ reselect fields poly class $=43$ \\ shadesymbol 96 \\ shadeput 543 \\ polygonshades fields 1000 \\ clearselect
}
/*select rye
reselect fields poly class $=44$
shadesymbol 89
shadeput 544
polygonshades fields 1000
clearselect

/ *select milo

reselect fields poly class $=45$

shadesymbol 99

shadeput 545

polygonshades fields 1000

clearselect

\title{
/*select Triticale
}

reselect fields poly class $=46$

shadesymbol 98

shadeput 546

polygonshades fields 1000

clearselect

/*select millet

reselect fields poly class $=47$

shadesymbol 101

shadeput 547

polygonshades fields 1000

clearselect

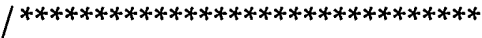

/ * FORAGE AND/OR PROGRAM ACRES

${ }^{*}$ *select hay (alfalfa, etc...) and forage and brome

reselect fields poly class $=51$

aselect fields poly class $=50$

aselect fields poly class $=52$

shadesymbol 71

shadeput 550 
polygonshades fields 1000

clearselect

/*select pasture

reselect fields poly class $=53$

shadesymbol 68

shadeput 553

polygonshades fields 1000

clearselect

/*select native grass

reselect fields poly class $=54$

shadesymbol 67

shadeput 554

polygonshades fields 1000

clearselect

/*select grass

reselect fields poly class $=55$

aselect fields poly class $=59$

shadesymbol 75

shadeput 555

polygonshades fields 1000

clearselect

/*select acr / crp

reselect fields poly class $=56$

aselect fields poly class $=57$

aselect fields poly class $=58$

shadesymbol 73

shadeput 556

polygonshades fields 1000

clearselect

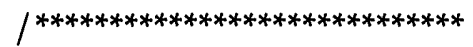

$I^{*}$ other crops

/*select sugar beets

reselect fields poly class $=60$

shadesymbol 118

shadeput 560

polygonshades fields 1000

clearselect

/ *select melons

reselect fields poly class $=61$

shadesymbol 72

shadeput 561

polygonshades fields 1000

clearselect

/*select orchards

reselect fields poly class $=62$ 
shadesymbol 124

shadeput 562

polygonshades fields 1000

clearselect

/*select garden

reselect fields poly class $=63$

shadesymbol 112

shadeput 563

polygonshades fields 1000

clearselect

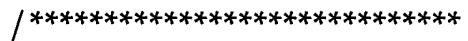

$1^{*}$ ROW CROPS

/*select corn

reselect fields poly class $=70$

shadesymbol 84

shadeput 570

polygonshades fields 1000

clearselect

${ }^{*}$ select beans

reselect fields poly class $=71$

shadesymbol 92

shadeput 571

polygonshades fields 1000

clearselect

/ *select sorgum

reselect fields poly class $=72$

shadesymbol 103

shadeput 572

polygonshades fields 1000

clearselect

/*select potatoes

shadeset colornames.shd

reselect fields poly class $=73$

shadesymbol 90

shadeput 573

polygonshades fields 1000

clearselect

$I^{*}$ select OTHER BEANS (pinto, kidney, green)

shadeset colornames.shd

reselect fields poly class $=74$

shadesymbol 62

shadeput 574

polygonshades fields 1000

clearselect 
/************************************

$I^{*}$ usage class

/*select non ag

reselect fields poly class $=98$

shadesymbol 107

shadeput 598

polygonshades fields 1000

clearselect

/*********************************

/* TILLAGE PRACTICE, ETC.

shadeset plotter.shd

/* double cropped

reselect fields poly double $=1$

shadesymbol 9

shadeput 600

polygonshades fields 1000

clearselect

$1 * * * * * * * * * * * * * * * * * * * * * * * * * * * * * * * *$

$l^{*}$ irrigated

reselect fields poly irrig $=1$

shadesymbol 21

shadeput 601

polygonshades fields 1000

clearselect

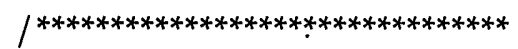

$I^{*}$ till

reselect fields poly till eq 1

shadesymbol 81

/*shadeput 602

polygonshades fields 1000

clearselect

reselect fields poly till eq 2

shadesymbol 77

/*shadeput 602

polygonshades fields 1000

clearselect

reselect fields poly till eq 3

shadesymbol 65

/*shadeput 602

polygonshades fields 1000

clearselect

reselect fields poly till eq 4 
shadesymbol 97

/*shadeput 602

polygonshades fields 1000

clearselect

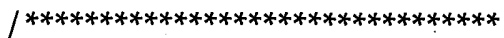

${ }^{*}$ cultivation practice

reselect fields poly cult ne 0

shadesymbol 65

shadeput 603

polygonshades fields 1000

clearselect

$1 * * * * * * * * * * * * * * * * * * * * * * * * * * * * * * * *$

$l^{*}$ failed crop

reselect fields poly fail $=1$

shadesymbol 65

shadeput 604

polygonshades fields 1000

clearselect

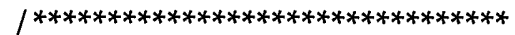

${ }^{*}$ highly erodable

reselect fields poly erode $=1$

shadesymbol 65

shadeput 605

polygonshades fields 1000

clearselect

\&return

\section{None.aml}

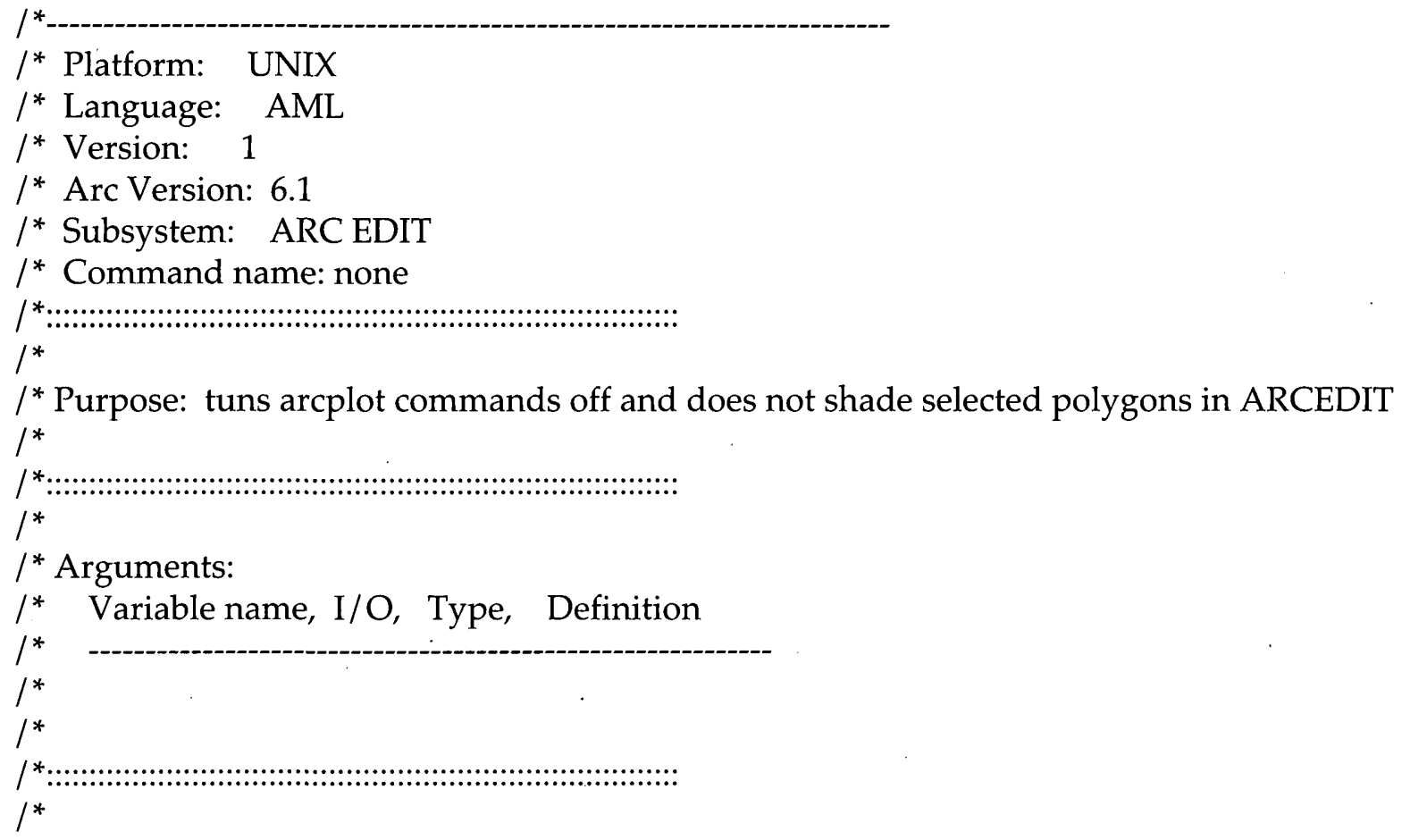


/ Programs or Menus called:

$1^{*}$

$1^{*}$

$1^{*}$

$I^{*}$ Programs or Menus called by: none

$1^{*}$

$1^{*}:$

/* History:

/* Author/Site, Date, Rev Event

1* - -------

/* Craig Harvey/USGS, Iowa 20 August 941 initial code

$1^{*}$

$1^{*}$

$1 *$

/* Disclaimer:

${ }^{*}$ Although this program had been used by the U. S. Geological Survey,

$/{ }^{*}$ no warranty, expressed or implied, is made by the USGS as to the accuracy

${ }^{*}$ and functionality of the program and related program material nor shall the fact

${ }^{*}$ of distribution constitute any such warranty, and no responsibility is assumed

/ by the USGS in connection herewith.

$1 *:$

ap none

images off

\&return

\section{Cprocess.aml}

$l^{*}$

/* Platform: UNIX

* Language: AML

/* Version: 3.2

/* Arc Version: 6.1

/* Subsystem: ARC

/ Command name: cprocess

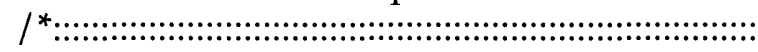

$l^{*}$

$I^{*}$ Purpose: To rectify and convert tif files to rgb grids, calc zero values in

$l^{*}$ those grids to NODATA, set merge precedence and merge to three grids,

$I^{*} \quad$ one each of the red/green/blue spectrum.

$1 *$

$1 *$

$1^{*}$

$l^{*}$ Arguments:

/* Variable name, I/O, Type, Definition

$l^{*}$

/* dir_name,

${ }^{*} \quad$.mrge_lst1

$/^{*}$.mrge_lst2

location of tif files under/images

merge list for red spectrum

merge list for green spectrum 


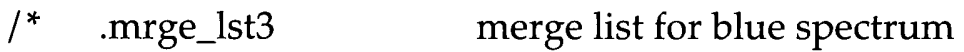

$I^{*} \quad$ alphanumeric letters represent tiff image names

$I^{*} \quad$ all variables ending in $\mathrm{c} 1, \mathrm{c} 2$ or $\mathrm{c} 3$ are intermediate process variables

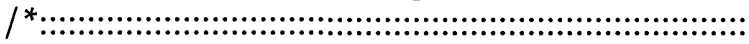

$1^{*}$

/* Programs or Menus called: GRID

$1^{*}$

$1^{*}$

$I^{*}$ Programs or Menus called by: none

$1^{*}$

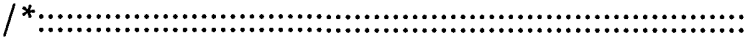

/* History:

/* Author/Site, Date, Rev Event

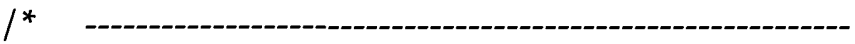

/* Craig Harvey/USGS, Iowa 15 June 941 initial code

/* Craig Harvey/USGS, Iowa 12 July 942 added nodata element

/* Craig Harvey/USGS, Iowa 20 July 943 added site name variable

1* Craig Harvey/USGS, Iowa 15 August 943.1 added variable list

I* Craig Harvey/USGS, Iowa 20 August 943.2 added merge list

$1^{*}$

$1^{*}$

$1 *$

I* Disclaimer:

${ }^{*}$ Although this program had been used by the U. S. Geological Survey,

I*no warranty, expressed or implied, is made by the USGS as to the accuracy

$/ *$ and functioning of the program and related program material nor shall the fact

$1^{*}$ of distribution constitute any such warranty, and no responsibilities assumed

/ ${ }^{*}$ by the USGS in connection herewith.

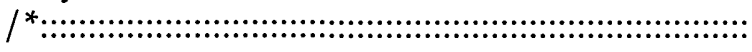

$I^{*}$ variable used to add items

$l^{*}$ This variable is the site name / directory name in images

\&sv dir_name ia18

$l^{*}$ variable list for loops during processing

${ }^{*}$ Delete those letters not needed

\&sv var_list a b c d f g

$I^{*}$ variables used to set order of precedence in merging and to create the

$I^{*}$ add the variables from above in order of precedence best to worst

$l^{*}$ set unused variables equal to zero.

\&sv $a=c$

$\& s v b=d$

$\& s v c=a$

$\& s v d=b$

\&sv $\mathrm{f}=\mathrm{f}$

$\& s v g=g$

$\& s v \mathrm{~h}=0$

$\& s v i=0$ 
$I^{*}$ merge_list variable

$I^{*}$ use zero to stop collection of merge variable.

${ }^{*}$ zero stop set from variable list above

\&sv .mrge_lst1 [before a\%a\%c1,a\%b\%c1,a\%c\%c1,a\%d\%c1,a\%f $\% c 1, a \% g \% c 1, a \% h \% c 1, a 0]$ \&sv .mrge_lst2 [before a\%a \%c2,a\%b\%c2,a\%c\%c2,a\%d\%c2,a\%f\%c2,a\%g\%c2,a\%h\%c2,a0] \&sv .mrge_lst3 [before a\%a\%c3,a\%b\%c3,a\%c\%c3,a\%d\%c3,a\%f\%c3,a\%g\%c3,a\%h\%c3 ,a0]

/ ${ }^{*}$ rectify images

\&do $\mathrm{k} \&$ \&list \%var_list $\%$

rectify $\% \mathrm{k} \%$.tif $\% \mathrm{k} \% \mathrm{k} \%$.tif

\&end

$I^{*}$ convert images to grids

\&do j \&list \%var_list\%

imagegrid \%j\%\% $\%$.tif \% $\%$

\&end

grid

setwindow $\%$ j\%1c1

setcell $\% \mathrm{j} \% \mathrm{c} 1$

setmask \%j\%c1

$I^{*}$ calculate zero cells to NODATA in preparation for merging

\&do i \&list \%var_list $\%$

setwindow \%i\%c1

setcell $\% \mathrm{i} \% \mathrm{cl}$

setmask off

$\mathrm{a} \% \mathrm{i} \% \mathrm{cl}=$ setnull $(\% \mathrm{i} \% \mathrm{cl}==0, \% \mathrm{i} \% \mathrm{c} 1)$

$\mathrm{a} \% \mathrm{i} \% \mathrm{c} 2=\operatorname{setnull}(\% \mathrm{i} \% \mathrm{c} 2==0, \% \mathrm{i} \% \mathrm{c} 2)$

$\mathrm{a} \% \mathrm{i} \% \mathrm{c3}=\operatorname{setnull}(\% \mathrm{i} \% \mathrm{c} 3==0, \% \mathrm{i} \% \mathrm{c} 3)$

\&end

$I^{*}$ merge the grids

setmask off

setwindow maxof

setcell minof

compc1 $=$ merge $(\%$. mrge_lst $1 \%)$

compc $2=$ merge $\left(\% . m r g e \_l s t 2 \%\right)$

compc3 $=$ merge $\left(\% . m r g e \_l s t 3 \%\right)$

quit

${ }^{*}$ create road buffer and append coverages together for field delineation

buffer \%dir_name\%rds rdsbuf \# \# 12 \# line

append fld 1 notest features

\%dir_name\%str

rdsbuf 
wellbuf

end

y

y

clean fld1

clip fld1 wellbuf fld2 poly

clean fld2

$I^{*}$ add items to pat and aat for field delineation rename fld 2 fields

additem fields.pat fields.pat (!double irrig till cult fail erode !) $11 \mathrm{i}$

additem fields.pat fields.pat class $412 \mathrm{f} 0$

build \%dir_name\%str line

additem \%dir_name\%str.aat \%dir_name\%str.aat major $11 \mathrm{i}$

\&return

$1 *$ display the grid

initarc 99993

grid

mape wellbuf

gridc rgb compc1 compc2 compc3

linecolor 3

arcs fields

\section{Dig.aml}

$1 *$

* Platform: UNIX

/* Language: AML

/* Version: 1.2

/* Arc Version: 6.1

/* Subsystem: ARC

/* Command name: dig.aml

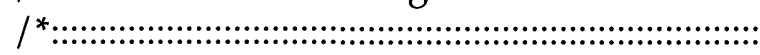

$I^{*}$ Uniformly sets up the editing environment for heads-up

${ }^{*}$ digitizing of the field/crop boundaries.

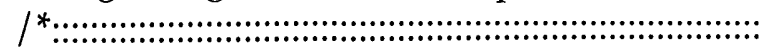

$I^{*}$ Arguments:

/* Variable name, I/O, Type, Definition

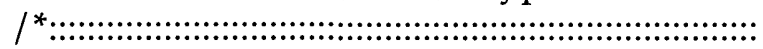

/* Programs or Menus called:

/ Programs or Menus called by: none

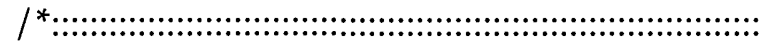

/* History:

/* Author/Site, Date, Rev Event

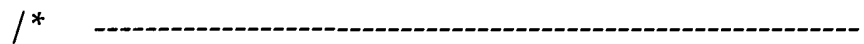

/ Craig Harvey/USGS, Iowa 15 June 941 initial code

$1 *$

/* Disclaimer: 
$I^{*}$ Although this program had been used by the U. S. Geological Survey, $I^{*}$ no warranty, expressed or implied, is made by the USGS as to the accuracy $I^{*}$ and functioning of the program and related program material nor shall the fact ${ }^{*}$ of distribution constitute any such warranty, and no responsibilities assumed $1{ }^{*}$ by the USGS in connection herewith.

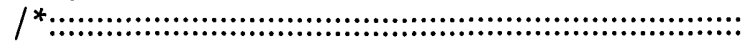

$l^{*}$ set edit and draw environments

mape fields

ec fields

de arcs node errors

nodecolor dangle 2

nodecolor pseudo 3

intersectarcs all

nodesnap closest 10

snapc fields

snapf arc arc

snapping closest 10

image comp

image off

bc wellbuf 3

be arcs

ef arcs

draw

\&return 


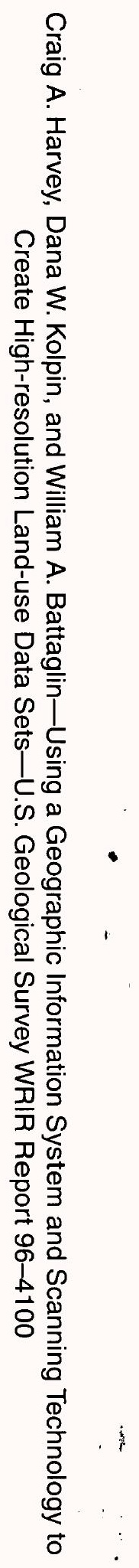

